\title{
RESEARCH
}

Open Access

\section{Growth, yield, genetic parameters and random amplified polymorphic DNA (RAPD) of five rice varieties treated with sodium azide and sown under different saline conditions}

\author{
Beckley Ikhajiagbe* (iD and Ujomonigho Edomo Omoregie
}

\begin{abstract}
Background: Rice is probably one of the most indispensable grain diets for the greater part of the world. It can be grown successfully under a wide range of climatic conditions; however, yields can be significantly diminished under moderate saline levels. Therefore, the employment of plant breeding techniques in enhancing plant survival and performance capacities under saline conditions becomes imperative. Mutation perhaps is one of the ultimate sources from which heritable variations could be selected from and hence are a useful tool to create raw materials for genetic improvement of rice. The effects of sodium azide $\left(\mathrm{NaN}_{3}\right)$, as a chemical mutagen, on the growth, yield, genetic parameters, and RAPD profile of five rice varieties, FARO-44, FARO-52, FARO-57, NERICA L-34, and NERICA L47 , sown in soils maintained at a conductivity range of 4000 to $6000 \mu \mathrm{s} / \mathrm{cm}$ using sodium chloride solutions were investigated. The planting materials were treated with $0 \% \mathrm{NaN}_{3}$ at $\mathrm{pH} 7$ (control) and a concentration range of 0 to $0.032 \% \mathrm{NaN}_{3}$ at $\mathrm{pH} 3$ for $6 \mathrm{~h}$.
\end{abstract}

Results: Sodium azide treatment significantly reversed the negative effects associated with plant development under saline conditions. Consequently, $\mathrm{NaN}_{3}$ treatment significantly improved growth, yield, and genetic parameters of the rice varieties under high salinity compared with the controls. High heritability values were recorded for number of tillers per plant (78.06\%), number of panicles per plant (78.74\%), and 100-grain weight (98.15\%) indicating the possibility of evolving higher yield variants through selection. Chlorophyll mutations observed were albino, viridis, and striata. The RAPD analyses with primers OPR 02 (5'-CAC AGC TGC C-3'), OPC 04 (5'-CCG CAT CTA C-3'), and OPC 05 (5'-GAT GAC CGC C-3') revealed different DNA banding patterns between controls and $\mathrm{NaN}_{3}$-treated plants.

*Correspondence: beckley.ikhajiagbe@uniben.edu; http://livedna.net/profile. php?dna=

Department of Plant Biology and Biotechnology, University of Benin, Benin, Nigeria

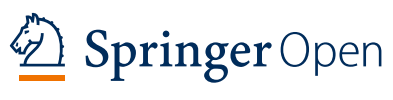

(c) The Author(s). 2020 Open Access This article is licensed under a Creative Commons Attribution 4.0 International License, which permits use, sharing, adaptation, distribution and reproduction in any medium or format, as long as you give appropriate credit to the original author(s) and the source, provide a link to the Creative Commons licence, and indicate if changes were made. The images or other third party material in this article are included in the article's Creative Commons licence, unless indicated otherwise in a credit line to the material. If material is not included in the article's Creative Commons licence and your intended use is not permitted by statutory regulation or exceeds the permitted use, you will need to obtain permission directly from the copyright holder. To view a copy of this licence, visit http://creativecommons.org/licenses/by/4.0/. 


\begin{abstract}
(Continued from previous page)
Conclusions: The study indicates that sodium azide is a potent mutagen, evident from information on genetic variability expressed by the DNA profiles of the rice varieties and their mutant counterparts as well as in the yield performances. The study thus indicated that the source of $\mathrm{NaN}_{3}$ induced variants among the treated progenies were genetic in nature and hence heritable. However, further screening on promising mutant plants from this research should be done through successive generations to identify stable traits and variant that can perform optimally under saline conditions.
\end{abstract}

Keywords: Genetic advance, Germination, Heritability, Oryza sativa, RAPD, Sodium azide

\section{Introduction}

Rice is a staple grain nourishment for an enormous piece of the total populace, making it the second most devoured cereal grain (Crayford and Shen 1998). As a customary nourishment plant in Africa, rice can improve nutritional sustenance, help nourishment security, cultivate rustic advancement, and bolster up economical land care. Rice gives more than one-fifth of the calories devoured worldwide by human (Heong et al. 2005). In mid-2008, a few governments and retailers started apportioning providers of the grain because of the dread of a worldwide rice deficiency (International Rice Research Institute 2008). More than sixty species of Oryza have been reported, but only twenty-three valid species are generally recognized. Cultivated rice does not cross freely with most wild species except that Oryza sativa and Oryza glaberrima intercross freely. It has been said that over eight thousand (8000) botanically different varieties of rice are in existence in the world. At present time, over eighteen thousand $(18,000)$ varieties are maintained in the world rice collected of the International Rice Research Institute (IRRI) (Juliano and Bechtel 1985).

Rice can be grown successfully under a wide range of climatic conditions. It generally requires a minimum growing season of 4 to 5 months, during which the mean temperature must average $21{ }^{\circ} \mathrm{C}$. Although rice culture extends from $45^{\circ} \mathrm{N}$ to $40^{\circ} \mathrm{S}$ latitude, most of the world rice crop is grown between the $40^{\circ} \mathrm{N}$ and $40^{\circ} \mathrm{S}$ latitudes. The crop is commonly associated with tropical regions, but it is also widely grown in temperate areas. Rice is grown at an altitude from sea level to over $1524 \mathrm{~m}$. Rice is described by pliancy which permits it to develop in practically any biophysical condition in the west and central Africa. Along these lines, rice is developed in an entire scope of agro-biological zones from the humid forest to the Sahel. Inside these local agro-natural zones, five fundamental rice-based frameworks can be recognized concerning water supply and geology in subSaharan Africa (SSA) (Windmeijer et al. 1994). These incorporate rain-sustained upland rice in levels and inclines; rain-sustained rice in valley base and flood fields with changing degrees of water control; inundated rice with generally great water control in deltas and flood fields; profound water, floating rice along riverbeds banks. Also, mangrove swamp rice in tidal ponds and deltas on beach front zones.

To overcome any issues among utilization and creation, Africa spends about US \$ 1.5 billion yearly on rice imports. The greatest concern is that the importation of rice is putting an expanding overwhelming interest on rare foreign currency reserve of the nations of this locale which are among the poorest on the planet (Nwanze et al. 2006). Only recently, the Federal Government of Nigeria, in bid to discourage rice imports and enhance local cultivation of rice, it shut its borders.

The Nigerian mangrove ecology has potential for one million hectares of cultivatable area for rice, but at present less than 100 ha are being developed. The ecology contributes less than $2 \%$ to national rice production with low rice yields of only about 1 tonne per hectare. Of about fifty-eight varieties that have been bred, only five are suited to the mangrove swamp ecological zone in Nigeria (Guei and Fagade 2002). Development of appropriate technology to expand and increase rice productivity in the mangrove swamp ecology is, therefore, the most urgent issue needing attention (Singh et al. 1997). Enormous zones of saline soils and slightly salty water areas are under-used worldwide for cropping (Oputa 1981). In the Niger Delta territory of Southern Nigeria (around $70,000 \mathrm{~km}^{2}$ ), around $30 \%$ of the soil are saline with electrical conductivities more than $0.5 \mathrm{~ms} / \mathrm{cm}$ and thus ineffective. Watered areas in the north of the nation are likewise progressively getting saltier due to overly application of fertilizer and may before long be confronted with a similar saltiness issue. One of the approaches to address this issue is through the identification and planting of salt tolerant crop varieties in these apparently ineffective natural surroundings (Mensah et al. 2006).

Rice yield can be diminished by up to $50 \%$ when developed under moderate saline levels. It is appraised as a salt sensitive variety (Shannon et al. 1998). Despite the fact that salinity influences all phases of the development of rice plant and the crop reactions to salinity differs with development stages, concentration, and length of 
presentation to the saline condition. In the most normally grown rice cvs., new seedlings were exceptionally salt sensitive (Lutts et al. 1995).

Soil salinity is a challenge to rice development under artificially watered agricultural system because of low quality of available water (Abdullah and Ahmed 1982). Rice is the significant crop of numerous waterfront areas which are inclined to the ocean water inflow during elevated tide and of rain-sustained frameworks which experience expanding saltiness in the root zone because of a rising water table. Soil salinity coming about because of common procedures from irrigation with saline water happens in numerous dry to semi dry districts of the world (Lauchli and Epstein 1990). As indicated by Tanji (Tanji 1990), 20\% of developed land overall is unfavorably influenced by high salt fixation which hinders plant development and yield. In warm and dry regions, salt fixation increments in the upper soil layer because of high rate of evaporation which surpasses precipitation (Ebert et al. 2002). Beating salt stress is a fundamental issue in these areas to guarantee the continuity of agricultural practices. Reproducing for salinity tolerance in rice is a challenge because of the contribution of a few genes controlling the character and absence of adequate information on the components controlling salt tolerance (Akita and Cabuslay 1988; Yeo et al. 1990). Accordingly, endeavors have been made to build up salt tolerance in some varieties through induced mutation.

Mutations are useful in contemplating the nature and capacity of genes which are the structural unit and basis of plant development, along these lines creating raw materials for the hereditary improvement of monetary yields (Adamu and Aliyu 2007). This method has been utilized to create numerous cultivars with improved economic worth and investigation of hereditary qualities and the miseries of plant development (Van et al. 1990; Bertagne-Sagnard et al. 1996). Different mutagenic agents are utilized to instigate positive changes at a high recurrence that incorporate ionizing radiation and chemical mutagens (Ahloowalia and Maluszynski 2001). Chemical mutagens produce induced mutation which leads to base pair substitutions, particularly GC $\rightarrow$ AT bringing about amino acid changes, which change the capacity of proteins yet do not abrogate their capacities as deletion and frameshift mutations for the most part do (Van der Veen 1966).

Some researchers have found chemical mutagens to be more effective than physical ones (Rao and Roa 1983; Dhanayanth and Reddy 2000; Bhat et al. 2005), while others found the reverse case (Tara and Dnyansager 1980; Zeerak 1991). Sodium azide (NaN3) is known to be exceptionally mutagenic in a few life forms including plants and creatures (Rines 1995; Veleminsky and Anglis 1987; Owais and Kleinhofs 1988; Raicu and Mixich
1992; Grant and Salamone 1994; Zhang and Hodson 2001), and its mutagenic potential has been accounted for in a few screening measures.

Seeds have high regenerative potential and are favorable for use in mutagenesis. In vitro method can be utilized for both seed and vegetatively cultivated species. The promutagen NaN3 is exceptionally utilized with seeds to induce mutations, which must be processed by plant cells to make mutagenic agent, probable azidoalanine (Owais et al. 1983). Azide likewise prompts high frequencies of chlorophyll and morphological transformations with an insignificant recurrence of chromosomal deviations when utilized in acidic conditions. Various different specialists (Mensah et al. 2006; Coe and Neuffer 1977; Mashenkov 1986; Mshembula et al. 2012; Omoregie et al. 2012; Ikhajiagbe et al. 2013; Ikhajiagbe and Oshomoh 2014; Ikhajiagbe and Akendolor 2016; Ikhajiagbe and Anoliefo 2017) have recently announced the part of chemical mutagens in improving hereditary variability in higher plants. Most of these workers used sodium in enhancing plant capacities.

The aim of the study is to investigate possible enhancement of growth, yield, and genetic parameters of five rice varieties treated with sodium azide and sown in salt-treated soil. Rice varieties selected for this study were improved lowland and shallow swamp highyielding varieties, already in cultivation by farmers in Nigeria (Dalton and Guei 2003; Akinwale et al. 2012; Maji 2009; Ojehomon et al. 2009). These were three FARO varieties and two NERICA varieties. Lowland and shallow swamp varieties were selected because part of the intention of the study was to screen the varieties and their sodium azide treated counterparts for tolerance to salinity by measuring the genetic distance and genetic parameters among them. This treatment will be useful in breeding program to improve the productivity of rice under saline conditions in the future.

\section{Materials and methods}

Three improved high yielding rice varieties, FARO-44, FARO-52, and FARO-57, and two lowlands NERICA varieties, L-34 and L-47, were obtained from the National Cereals Research Institute, Badeggi, Bida, Niger State. The experiment was conducted at the Department of Plant Biology and Biotechnology Screen House, located beside the Faculty of Life Sciences, University of Benin, Nigeria. Top soil (0-10-cm depth) which physicochemical parameters were determined in the Laboratory prior to use (Table 1) was collected from the fallow site attached to the Botanic garden of the Department of Plant Biology and Biotechnology, University of Benin. Soil was sun-dried to constant weight before measured into experiment bowls. A sample of this pooled soil was sent to the laboratory for physicochemical analysis prior 
Table 1 Physicochemical properties of planting soil

\begin{tabular}{|c|c|c|}
\hline Parameters & Units & Mean \\
\hline $\mathrm{pH}$ & - & 6.02 \\
\hline Electric conductivity & $\mu \mathrm{s} / \mathrm{cm}$ & 303 \\
\hline Total organic carbon & $\%$ & 0.41 \\
\hline Total nitrogen & $\%$ & 0.13 \\
\hline Exchangeable acidity & meq/100 g soil & 0.2 \\
\hline $\mathrm{Na}^{+}$ & meq/100 g soil & 11.46 \\
\hline $\mathrm{K}^{+}$ & meq/100 g soil & 1.54 \\
\hline $\mathrm{Ca}^{2+}$ & meq/100 g soil & 15.6 \\
\hline $\mathrm{Mg}^{2+}$ & meq/100 g soil & 11.3 \\
\hline $\mathrm{Cl}^{-}$ & $\mathrm{mg} / \mathrm{l}$ & 1666 \\
\hline Available phosphates & $\mathrm{mg} / \mathrm{l}$ & 153 \\
\hline Ammonium nitrogen & $\mathrm{mg} / \mathrm{l}$ & 25.4 \\
\hline Nitrite & $\mathrm{mg} / \mathrm{l}$ & 15.01 \\
\hline Nitrate & $\mathrm{mg} / \mathrm{l}$ & 30.75 \\
\hline Sulfates & $\mathrm{mg} / \mathrm{l}$ & 14.63 \\
\hline Clay & $\%$ & 4.4 \\
\hline Silt & $\%$ & 7.8 \\
\hline Sand & $\%$ & 87.8 \\
\hline $\mathrm{Fe}^{3+}$ & mg/l & 1011.43 \\
\hline $\mathrm{Mn}^{2+}$ & $\mathrm{mg} / \mathrm{l}$ & 17.03 \\
\hline $\mathrm{Zn}^{2+}$ & $\mathrm{mg} / \mathrm{l}$ & 14.76 \\
\hline $\mathrm{Cu}^{2+}$ & mg/l & 3.31 \\
\hline $\mathrm{Cr}^{+}$ & $\mathrm{mg} / \mathrm{l}$ & 2.11 \\
\hline $\mathrm{Cd}^{+}$ & $\mathrm{mg} / \mathrm{l}$ & $>0.001$ \\
\hline $\mathrm{Pb}^{2+}$ & $\mathrm{mg} / \mathrm{l}$ & 0.03 \\
\hline $\mathrm{Ni}^{+}$ & $\mathrm{mg} / \mathrm{l}$ & 2.54 \\
\hline $\mathrm{V}^{+}$ & $\mathrm{mg} / \mathrm{l}$ & 1.36 \\
\hline Total hydrocarbon content & $\mathrm{mg} / \mathrm{l}$ & 754 \\
\hline
\end{tabular}

to use in the experiment. The results of the analysis have been presented in Table 1.

\section{Analysis of physicochemical parameters}

Physicochemical parameters of soil were determined prior to usage of the soil according to standard procedures. In other to determine soil $\mathrm{pH}$ and soil conductivity, $20 \mathrm{~g}$ of air-dried soil was sieved. Estimated $20 \mathrm{ml}$ of distilled water was then added to it and left for $30 \mathrm{~min}$. The mixture was mixed once in a while with a glass rod. Soil $\mathrm{pH}$ was measured by utilizing a $\mathrm{pH}$ meter (Model PHS-3C), and the soil conductivity read through a handheld conductivity meter (HI 70039P, Hanna Instruments). Soil total organic carbon (TOC) was determined following the methods of (Nelson and Sommers 1982). Determination of other physicochemical parameters followed the methods of (AOAC (Association of Official Analytical Chemists) 2005; Osuji and Nwoye 2007; El-
Sharabasy and Ibrahim 2010). For determination of total hydrocarbons, $5 \mathrm{~g}$ soil was weighed into a $100-\mathrm{ml}$ plastic bottle. Afterwards, $25 \mathrm{ml}$ of $\mathrm{n}$-Hexane was included and precisely shaken for $10 \mathrm{~min}$ and let stand secured. It was then separated, and the filtrate was perused at $460 \mathrm{~nm}$. THC standard stock, $1000 \mathrm{mg} / \mathrm{l}$, was done by pipetting some $1.18 \mathrm{ml}$ of Forcados Blend Crude Oil and made to 11 with n-Hexane. From this, 0, 10, 20, 40, 60, 80, and $100 \mathrm{mg} / \mathrm{l}$ working models were made ready.

Calculation:

$$
\mathrm{THC}(\mathrm{mg} / \mathrm{l})=\frac{\text { Instr.reading } \times \text { Slope reciprocal } \times 25}{5 \mathrm{~g}}
$$

The experimental design was a completely randomized design with four blocks. Each block was a replicate of five rice varieties and six treatment levels including control. There were 30 perforated pots per block and a total of 120 pots for the experiment.

\section{Preparation of sodium azide $\left(\mathrm{NaN}_{3}\right)$ solutions}

The first set of grains was prepared and used to test for the $\mathrm{LD}_{50} . \mathrm{NaN}_{3}$ concentrations of $1 \%, 0.25 \%, 0.0625 \%$, and $0.032 \%$ were prepared by weighing $1 \mathrm{~g}, 0.25 \mathrm{~g}$, $0.0625 \mathrm{~g}$, and $0.032 \mathrm{~g}$, respectively, and dissolving each in $100 \mathrm{ml}$ of distilled water pre-directed to $\mathrm{pH} 3$ with ortho-phosphate. After LD50 was established, decreased concentrations of $0.032 \%, 0.016 \%, 0.008 \%$, and $0.004 \%$ $\mathrm{NaN} 3$ were set up by estimating proper volumes from stock solution and making up to $100 \mathrm{ml}$ with orthophosphoric acid managed water $(\mathrm{pH} 3)$. Zero percent level was water at $\mathrm{pH} 3$ with no NaN3. Control was just distilled water at $\mathrm{pH} 7$.

\section{Preparation of sodium chloride $(\mathrm{NaCl})$ solution}

Sodium chloride solutions of conductivities within the range of 4000 to $6000 \mu \mathrm{s} / \mathrm{cm}$, estimated as moderate salinity, were prepared. Conductivity was read using DiST Conductivity/TDS meter (HI 70039P, Hanna Instruments). Conductivity of soil was monitored throughout the course of research. This was achieved by measuring soil solutions of 1:2 soil/water proportions. Conductivity of $\mathrm{NaCl}$-treated soil was always at least a double fold of control.

\section{Grain treatments}

Healthy uniform grains were chosen by hand-picking and moved into named Petri-dishes. Grains were allowed to pre-absorbed tap water for $14 \mathrm{~h}$ and afterward moved to NaN3 ( $\mathrm{pH} 3$ ) solution for $6 \mathrm{~h}$ with constant mixing. Toward the finish of the introduction to NaN3 treatment, grains were appropriately rinsed with water. 


\section{Sowing}

Grains were sown. Twenty grains were planted per pot.

\section{Watering and application of salt-treatment}

Plants were watered daily to avoid dehydration since the experiment was conducted in a screen house. Two hundred milliliters of $\mathrm{NaCl}$ solutions were applied once a week on all treatment levels except control.

\section{Fertilizer application}

Organic (composted farm yard manure) was applied twice; first at the fourth week after planting (4 WAP), then at the eighth week after planting (8 WAP). Inorganic fertilizer (N.P.K. 15:15:15) was applied at the twelfth week after planting, the onset of flowering, to argument high nutrient demand at that phase. Application was at the rate of $4 \mathrm{~g} / \mathrm{kg}$ (Ikhajiagbe et al. 2009). The fertilizer was first dissolved in water and applied at $5 \mathrm{~cm}$ away from the perimeter of the plants to avoid scotching of the plants.

\section{Percentage emergence}

The number of plants that emerged from the soil per pot was counted daily until 14 DAP.

Percentage emergence was then calculated by:

Number of grains emerging $\times 100$

Total number of grains sown

\section{Other plant growth measurements}

Plant heights were measured once a week from the third week after planting (WAP) to 5 WAP, then fortnightly from 7 WAP to 11 WAP using a measuring tape. The day the first panicle emerged in a particular treatment level of a variety was recorded as the number of days to flowering of that plant. The days of emergence of subsequent panicles were also recorded until 118 DAP.

The number of tillers per plant was counted after more than $50 \%$ of all the varieties have flowered (120 DAP). A number of panicles per plant were counted at the time of harvest. Panicles were harvested when they turned straw color (Nwilene et al. 2008).

One hundred grains per plant were weighed from the harvested grains. The number of plants that lodged per variety was counted, and their percentages calculated by as follows:

Percentage lodging:

Number of plants that logde $\times 100$

Total number of plants

For biomass, whole plants left until they turned straw color in the screen house were carefully uprooted and weighed.

\section{Chlorophyll content index (CCI)}

The chlorophyll content index of leaves of plants was estimated at 5 WAP during the vegetative stage and 12 WAP at the beginning of blooming, utilizing a chlorophyll content meter, CCM-200 plus. CCM-200 is a nondamaging chlorophyll content estimating meter. The normal meter perusing of five leaves for each plant was taken as the chlorophyll content index (CCI). Chlorophyll has a few particular optical absorbance attributes that the CCM-200 plus endeavors so as to decide relative chlorophyll concentration. Chlorophyll transmission is naturally high close to the infrared range and low in the red range since green plants absorb visible radiation for photosynthesis and transmit close to infrared, which they do not utilize. Strong absorbance bands are available in the blue and red yet not in the green or infrared bands. The CCM-200 or more uses absorbance to assess the chlorophyll content in leaf tissues. It utilizes LEDs that produces explicit wavelengths in the red and infrared reaches. Two wavelengths are utilized for absorbance measurement. One wavelength falls inside the chlorophyll absorbance range while the second serves to make up for mechanical contrasts, for example, tissue thickness. The detector uses the absorbance proportion of the two wavelengths to figure out a CCI esteem that is relative to the measure of chlorophyll in the sample (Apogee Instruments Incorporated n.d.).

Genetic parameters considered in the study include the following (Johnson et al. 1955; Allard 1999):

Phenotypic variance $\left(\delta^{2} \mathrm{ph}\right)$ : treatment mean square (TMS) between means

Genotypic variance $\left(\delta^{2} \mathrm{~g}\right)$ : difference between treatment mean square and error mean square (EMS)

Heritability (H\%):

$$
\frac{\delta 2 \mathrm{~g} \times 100}{\delta 2 \mathrm{ph}}
$$

Genetic advance (GA):

$$
\frac{\delta 2 \mathrm{~g} \times \mathrm{K}}{\sqrt{(\delta 2 \mathrm{ph})}}
$$

where $K=2.06$ (selection differential at 10\%).

Genetic gain (GG\%):

$$
\frac{\mathrm{GA} \times 100}{\text { Mean }}
$$

The genetic gain (GG\%) was calculated in terms of the genetic advance (GA) expressed as a percentage of the population mean $(X)$.

\section{Second generation $\left(\mathrm{M}_{2}\right)$ comparative studies}

Twenty one progenies made up of plants with the highest number of panicles per plant in each variety 
including their controls were selected and their seeds planted for $\mathrm{M}_{2}$. The means of percentage emergence and plant heights at 7 WAP were measured. Chlorophyll mutations were also observed. The spectrum of chlorophyll mutations was studied, and the mutants were classified as per the scheme of Gustafson (Gustafson 1940) with modifications by Lal et al. (Lal et al. 2009).

Albino-white, lethal, no chlorophyll, or carotenoids are formed.

Xantha-yellow to yellowish white, lethal, carotenoids present but chlorophyll absent.

Dark xantha-dark yellow, lethal.

Chlorina-uniform green color with white on tips, viable.

Viridis-uniform light yellow green color of leaves, viable.

Striata-longitudinal strips of different colors, viable.

\section{Random amplified polymorphic DNA (RAPD) studies DNA extraction}

DNA extraction from the leaf of the progenies of the Oryza sativa varieties and selected sodium azide treated counterparts was carried out using a ZR plant/seed DNA extraction kit according to the manufacturer's instruction. Briefly, $150 \mathrm{mg}$ of the rice leaf was added to a ZR BashingBead ${ }^{\mathrm{m}}$ containing $750 \mu \mathrm{l}$ of the lysis solution. The leaf was then homogenized using a mini bead beater for minutes. After homogenization, the tube was centrifuged at $10,000 \times g$ for $1 \mathrm{~min}$. Four hundred microliters of the supernatant was transferred into a Zymo-Spin ${ }^{\mathrm{Tn}}$ IV Spin Filter in a collection tube and centrifuged at 7000 rpm for $1 \mathrm{~min}$. One thousand two hundred microliters of Plant/Seed DNA Binding Buffer was then added to the filtrate in the collection tube. This mixture was transferred to a Zymo-Spin ${ }^{\text {Tx }}$ II Column in a collection tube and centrifuged at $10,000 \times g$ for $1 \mathrm{~min}$. The flow through from the collection tube was discarded, and $500 \mu \mathrm{l}$ of Plant/Seed DNA Wash Buffer was added to the Zymo-Spin $^{\text {tw }}$ II Column in a new collection tube and centrifuged at $10,000 \times g$ for $1 \mathrm{~min}$. After repeating the wash step, the column was transferred to a clean $1.5-\mathrm{ml}$ microcentrifuge tube, and $100 \mu \mathrm{l}$ of sterile water was added directly to the column matrix after which the tubes were centrifuged at $10,000 \times g$ for $30 \mathrm{~s}$ to elute the DNA. Finally, the eluted DNA was filtered using a Zymo-Spin $^{\text {ma }}$ IV-HRC Spin Filter into a 1.5 -ml microcentrifuge tube and centrifuged at $10,000 \times g$ for $30 \mathrm{~s}$.

\section{Polymerase Chain Reaction}

RAPD-PCR was carried out on the extracted DNA sample using the RAPD primer OPR $02\left(5^{\prime}-\mathrm{CAC}\right.$ AGC TGC C-3'), OPC 04 (5'-CCG CAT CTA C-3'), and OPC 05 (5'-GAT GAC CGC C-3'). The PCR reaction was carried out in a $20 \mu \mathrm{l}$ reaction mixture containing
1× PCR buffer (Solis Biodyne), $2.5 \mathrm{mM}$ magnesium chloride, $0.2 \mathrm{mM}$ of each dNTP, $40 \mathrm{pMol}$ of primer, $1 \mathrm{U}$ Taq DNA polymerase, and 10-200 ng of DNA, and sterile deionized water was used to make up the reaction mixture.

Amplification was carried out in an Eppendorf Nexus thermal cycler using the following cycling parameters: an initial denaturation at $95{ }^{\circ} \mathrm{C}$ for $5 \mathrm{~min}$ which was followed by 40 consecutive cycles of $95^{\circ} \mathrm{C}$ for $1 \mathrm{~min}, 30$ ${ }^{\circ} \mathrm{C}$ for $1 \mathrm{~min}$, and $72{ }^{\circ} \mathrm{C}$ for $2 \mathrm{~min}$. This was followed by a final extension of $72{ }^{\circ} \mathrm{C}$ for $10 \mathrm{~min}$. The PCR products were separated on a $1 \%$ agarose gel, and $1 \mathrm{~kb}$ DNA ladder (Fermentas) was used as DNA molecular weight standard.

\section{Statistical analysis}

Means and standard errors were calculated from data obtained. Data were analyzed following two-way analysis of variance using GENSTAT (8th edition). Where significant $F$-values were obtained, differences between means were separated using Student Newman Keuls test (Alika 2006).

\section{Results}

Table 2 showed the percentage emergence of the five rice varieties used for this study from day 2 after planting (2 DAP) to 14 DAP. At 2 DAP, FARO-44 had its highest emergence at $0 \% \mathrm{NaN}_{3}$ of $66.25 \%$. Emergence had not started at $0.016 \% \mathrm{NaN}_{3}$ and $0.032 \% \mathrm{NaN}_{3}$. Highest emergence at FARO-52 was also at $0 \% \mathrm{NaN}_{3}$ with $42.50 \%$ and no emergence at $0.032 \% \mathrm{NaN}_{3}$. FARO-57 had highest emergence at control and $0 \% \mathrm{NaN}_{3}$ with $31.25 \%$, respectively. There was no emergence at $0.032 \%$ $\mathrm{NaN}_{3}$. NERICA L-34 had the highest emergence at control with $70.00 \%$ and no emergence in $0.016 \% \mathrm{NaN}_{3}$ and $0.032 \% \mathrm{NaN}_{3}$, respectively. There was no emergence for all treatment levels control inclusive for NERICA L-47 at 2 DAP.

At 5 DAP, FARO- 44 and FARO-52 had $0 \% \mathrm{NaN}_{3}$ with the highest emergence of $96.25 \%$, respectively. $0.032 \%$ $\mathrm{NaN}_{3}$ of both varieties was also lowest with $26.25 \%$ and $58.75 \%$, respectively. FARO-57, NERICA L-34, and NERICA L-47 had their controls with the highest emergence of $96.25 \%, 98.75 \%$, and $11.25 \%$, respectively.

At 14 DAP, FARO- 44 had $97.50 \%$ as highest emergence in $0 \% \mathrm{NaN}_{3}$ and $0.016 \% \mathrm{NaN}_{3} .0 .004 \% \mathrm{NaN}_{3}$ of FARO-44 had lowest emergence of $90 \%$. FARO-52 had $100 \%$ emergence at $0 \% \mathrm{NaN}_{3}$ and $95 \%$ in all other levels except $0 \% \mathrm{NaN}_{3}$ which was $98.75 \%$. FARO-57 and NERICA L-34 had controls as highest emergence with 98.75\% each and $0.032 \% \mathrm{NaN}_{3}$ as lowest with $91.25 \%$ and $78.75 \%$, respectively. NERICA L-47 had control as highest emergence of $40 \%, 0.016 \% \mathrm{NaN}_{3}$ of $1.25 \%$, and no emergence for $0.032 \% \mathrm{NaN}_{3}$. 
Table 2 Percentage emergence of the five rice varieties

\begin{tabular}{|c|c|c|c|c|c|c|c|c|}
\hline \multirow[t]{2}{*}{ Variety } & \multirow{2}{*}{$\begin{array}{l}\text { NaN3 } \\
\text { treatment } \\
\text { level }\end{array}$} & \multicolumn{7}{|l|}{ Days after planting } \\
\hline & & Day 2 & Day 3 & Day 4 & Day 5 & Day 6 & Day 12 & Day 14 \\
\hline FARO-44 & Control & $52.50 \pm 5.20^{\mathrm{egh}}$ & $83.75 \pm 5.15^{\text {hij }}$ & $87.50 \pm 6.29^{e f}$ & $90.00 \pm 4.08^{d}$ & $91.25 \pm 4.27^{g h}$ & $91.25 \pm 4.27^{c}$ & $91.25 \pm 4.27^{c}$ \\
\hline FARO-44 & $0 \%$ & $66.25 \pm 2.39^{h}$ & $92.50 \pm 5.95^{\mathrm{ij}}$ & $95.00 \pm 3.54^{\mathrm{ef}}$ & $96.25 \pm 3.75^{d}$ & $97.50 \pm 2.50^{h}$ & $97.50 \pm 2.50^{c}$ & $97.50 \pm 2.50^{c}$ \\
\hline FARO-44 & $0.004 \%$ & $36.25 \pm 13.39^{\text {def }}$ & $83.75 \pm 1.25^{h i j}$ & $86.20 \pm 1.25^{\mathrm{ef}}$ & $88.75 \pm 1.25^{d}$ & $88.75 \pm 1.25^{g h}$ & $90.00 \pm 0^{c}$ & $90.00 \pm 0^{c}$ \\
\hline FARO-44 & $0.008 \%$ & $11.25 \pm 2.39^{\mathrm{abc}}$ & $73.75 \pm 2.89^{\text {hi }}$ & $87.50 \pm 3.23^{e f}$ & $91.25 \pm 2.39^{d}$ & $95.00 \pm 2.04^{g h}$ & $96.25 \pm 2.39^{c}$ & $96.25 \pm 2.39^{c}$ \\
\hline FARO-44 & $0.016 \%$ & $0.00^{\mathrm{a}}$ & $20.00 \pm 2.89^{\text {bcef }}$ & $47.50 \pm 6.61^{c d}$ & $77.50 \pm 3.23^{d}$ & $80.00 \pm 2.04^{\mathrm{fgh}}$ & $97.50 \pm 1.44^{c}$ & $97.50 \pm 1.44^{c}$ \\
\hline FARO-44 & $0.032 \%$ & $0.00^{\mathrm{a}}$ & $2.50 \pm 1.44^{\mathrm{abc}}$ & $13.70 \pm 5.54^{\mathrm{ab}}$ & $26.25 \pm 4.27^{b}$ & $42.50 \pm 4.38^{g h}$ & $91.25 \pm 5.15^{c}$ & $92.50 \pm 4.79^{c}$ \\
\hline FARO-52 & Control & $36.25 \pm 8.75^{\text {defg }}$ & $80.00 \pm 7.07^{\mathrm{hij}}$ & $90.00 \pm 2.89^{e f}$ & $92.50 \pm 3.23^{d}$ & $96.25 \pm 2.39^{g h}$ & $98.75 \pm 1.25^{c}$ & $98.75 \pm 1.25^{c}$ \\
\hline FARO-52 & $0 \%$ & $42.50 \pm 6.29^{\text {efgh }}$ & $83.75 \pm 6.25^{\text {hij }}$ & $88.80 \pm 5.15^{\mathrm{ef}}$ & $93.75 \pm 4.73^{d}$ & $98.75 \pm 1.25^{\mathrm{h}}$ & $100.00 \pm 0^{c}$ & $100.00 \pm 0^{c}$ \\
\hline FARO-52 & $0.004 \%$ & $20.00 \pm 4.56^{\mathrm{abcd}}$ & $83.75 \pm 9.87^{\text {hij }}$ & $87.50 \pm 2.23^{e f}$ & $91.25 \pm 5.91^{d}$ & $95.00 \pm 2.89^{g h}$ & $95.00 \pm 2.89^{c}$ & $95.00 \pm 2.89^{c}$ \\
\hline FARO-52 & $0.008 \%$ & $26.25 \pm 10.08^{\text {bcde }}$ & $83.75 \pm 6.25^{\text {hij }}$ & $87.50 \pm 4.33^{\mathrm{ef}}$ & $92.50 \pm 2.50^{d}$ & $92.50 \pm 2.50^{g h}$ & $95.00 \pm 2.89^{c}$ & $95.00 \pm 2.89^{c}$ \\
\hline FARO-52 & $0.016 \%$ & $2.50 \pm 1.44^{\mathrm{a}}$ & $21.25 \pm 5.54^{\mathrm{cf}}$ & $80.00 \pm 8.42^{e f}$ & $87.50 \pm 5.20^{d}$ & $92.50 \pm 2.23^{g h}$ & $95.00 \pm 3.54^{c}$ & $95.00 \pm 3.54^{c}$ \\
\hline FARO-52 & $0.032 \%$ & $0.00^{\mathrm{a}}$ & $2.50 \pm 1.44^{\mathrm{abc}}$ & $28.70 \pm 7.74^{\mathrm{abc}}$ & $58.75 \pm 2.39^{c}$ & $73.75 \pm 5.54^{\mathrm{efg}}$ & $95.00 \pm 5.00^{c}$ & $95.00 \pm 5.00^{c}$ \\
\hline FARO-57 & Control & $31.25 \pm 5.54^{\text {bcde }}$ & $78.75 \pm 4.73^{\text {hij }}$ & $86.20 \pm 5.54^{\mathrm{ef}}$ & $96.25 \pm 2.39^{d}$ & $98.75 \pm 1.25^{h}$ & $98.75 \pm 1.25^{c}$ & $98.75 \pm 1.25^{c}$ \\
\hline FARO-57 & $0 \%$ & $31.25 \pm 1.25^{\text {bde }}$ & $75.00 \pm 5.00^{h i}$ & $81.20 \pm 2.39^{e f}$ & $91.25 \pm 3.75^{d}$ & $92.50 \pm 4.33^{g h}$ & $96.25 \pm 2.39^{c}$ & $96.25 \pm 2.39^{c}$ \\
\hline FARO-57 & $0.004 \%$ & $12.50 \pm 3.75^{\mathrm{abc}}$ & $76.25 \pm 2.39^{h i}$ & $87.50 \pm 3.23^{e f}$ & $91.25 \pm 3.75^{d}$ & $92.50 \pm 3.23^{g h}$ & $96.25 \pm 2.39^{c}$ & $96.25 \pm 2.39^{c}$ \\
\hline FARO-57 & $0.008 \%$ & $3.75 \pm 3.75^{\mathrm{a}}$ & $51.25 \pm 4.27^{9}$ & $80.00 \pm 8.42^{\mathrm{ef}}$ & $93.75 \pm 2.39^{d}$ & $95.00 \pm 2.89^{g h}$ & $96.25 \pm 2.39^{c}$ & $96.25 \pm 2.39^{c}$ \\
\hline FARO-57 & $0.016 \%$ & $0.00^{\mathrm{a}}$ & $1.25 \pm 1.25^{\mathrm{ab}}$ & $26.20 \pm 4.27^{\mathrm{abc}}$ & $51.25 \pm 9.44^{c}$ & $60.00 \pm 9.13^{\mathrm{e}}$ & $91.25 \pm 3.75^{\complement}$ & $92.50 \pm 3.23^{c}$ \\
\hline FARO-57 & $0.032 \%$ & $1.25 \pm 1.25^{\mathrm{a}}$ & $1.25 \pm 1.25^{\mathrm{ab}}$ & $5.00 \pm 2.04^{\mathrm{ab}}$ & $31.25 \pm 4.27^{b}$ & $43.75 \pm 9.21^{d}$ & $91.25 \pm 4.27^{c}$ & $91.25 \pm 4.27^{c}$ \\
\hline NERICA L-34 & Control & $70.00 \pm 6.77^{h}$ & $97.50 \pm 1.44^{j}$ & $98.75 \pm 1.25^{\mathrm{f}}$ & $98.75 \pm 1.25^{d}$ & $98.75 \pm 1.25^{h}$ & $98.75 \pm 1.27^{c}$ & $98.75 \pm 1.27^{c}$ \\
\hline NERICA L-34 & $0 \%$ & $57.50 \pm 7.77^{g h}$ & $80.00 \pm 3.54^{\mathrm{hij}}$ & $65.00 \pm 22.17^{\mathrm{de}}$ & $90.00 \pm 4.56^{d}$ & $93.75 \pm 2.39^{g h}$ & $93.75 \pm 2.39^{c}$ & $93.75 \pm 2.39^{c}$ \\
\hline NERICA L-34 & $0.004 \%$ & $18.75 \pm 1.25^{\mathrm{ab}}$ & $82.50 \pm 5.20^{\text {hij }}$ & $88.50 \pm 4.73^{e f}$ & $88.75 \pm 5.54^{d}$ & $92.50 \pm 1.44^{g h}$ & $92.50 \pm 1.44^{c}$ & $92.50 \pm 1.44^{c}$ \\
\hline NERICA L-34 & $0.008 \%$ & $11.25 \pm 5.15^{\mathrm{ab}}$ & $65.00 \pm 8.17^{h}$ & $78.70 \pm 8.99^{\mathrm{ef}}$ & $83.75 \pm 5.54^{d}$ & $87.50 \pm 4.33^{g h}$ & $90.00 \pm 3.54^{c}$ & $90.00 \pm 3.54^{c}$ \\
\hline NERICA L-34 & $0.016 \%$ & $0.00^{\mathrm{a}}$ & $6.25 \pm 3.14^{\text {abcdef }}$ & $37.50 \pm 4.33^{c}$ & $56.25 \pm 8.26^{c}$ & $68.75 \pm 5.15^{\mathrm{ef}}$ & $91.25 \pm 4.27^{c}$ & $91.25 \pm 4.27^{c}$ \\
\hline NERICA L-34 & $0.032 \%$ & $0.00^{\mathrm{a}}$ & $0.00^{\mathrm{a}}$ & $5.00 \pm 3.54^{\mathrm{ab}}$ & $12.50 \pm 5.95^{\mathrm{a}}$ & $18.75 \pm 5.54^{\mathrm{abc}}$ & $78.75 \pm 5.15^{c}$ & $78.75 \pm 5.15^{c}$ \\
\hline NERICA L-47 & Control & $0.00^{\mathrm{a}}$ & $6.25 \pm 4.73^{\mathrm{abcdef}}$ & $11.20 \pm 4.73^{\mathrm{ab}}$ & $11.25 \pm 4.73^{\mathrm{a}}$ & $27.50 \pm 16.5^{c}$ & $40.00 \pm 20.9^{b}$ & $40.00 \pm 20.92^{b}$ \\
\hline NERICA L-47 & $0 \%$ & $0.00^{\mathrm{a}}$ & $3.75 \pm 2.39^{\text {abcde }}$ & $7.50 \pm 1.44^{\mathrm{ab}}$ & $7.50 \pm 1.44^{\mathrm{a}}$ & $7.50 \pm 1.44^{\mathrm{ab}}$ & $12.50 \pm 1.44^{\mathrm{a}}$ & $12.50 \pm 1.44^{\mathrm{a}}$ \\
\hline NERICA L-47 & $0.004 \%$ & $0.00^{\mathrm{a}}$ & $0.00^{\mathrm{a}}$ & $0.00^{\mathrm{a}}$ & $3.75 \pm 1.25^{\mathrm{a}}$ & $5.00 \pm 0^{\mathrm{ab}}$ & $8.75 \pm 2.39^{\mathrm{a}}$ & $8.75 \pm 2.39^{a}$ \\
\hline NERICA L-47 & $0.008 \%$ & $0.00^{a}$ & $0.00^{\mathrm{a}}$ & $0.00^{\mathrm{a}}$ & $0.00^{\mathrm{a}}$ & $0.00^{\mathrm{a}}$ & $6.25 \pm 2.39^{a}$ & $6.25 \pm 2.39^{a}$ \\
\hline NERICA L-47 & $0.016 \%$ & $0.00^{a}$ & $0.00^{\mathrm{a}}$ & $0.00^{\mathrm{a}}$ & $0.00^{\mathrm{a}}$ & $0.00^{\mathrm{a}}$ & $1.25 \pm 1.25^{\mathrm{a}}$ & $1.25 \pm 1.25^{\mathrm{a}}$ \\
\hline NERICA L-47 & $0.032 \%$ & $0.00^{\mathrm{a}}$ & $0.00^{\mathrm{a}}$ & $0.00^{\mathrm{a}}$ & $0.00^{\mathrm{a}}$ & $0.00^{a}$ & $0.00^{a}$ & $0.00^{\mathrm{a}}$ \\
\hline
\end{tabular}

Mean \pm standard error. Mean of four replicates. Different alphabets in columns indicate significant differences at $5 \%$ level of probability

Table 3 shows plant heights of the five varieties from 3 WAP to 11 WAP. At 3 WAP, plant heights of FARO-44 ranged from $32.88 \mathrm{~cm}$ in $0.032 \% \mathrm{NaN}_{3}$ to $36.00 \mathrm{~cm}$ in control. FARO-52 ranged from $29.52 \mathrm{~cm}$ in $0.032 \%$ $\mathrm{NaN}_{3}$ to $35.35 \mathrm{~cm}$ in control. FARO-57 ranged from $30.25 \mathrm{~cm}$ in $0.016 \% \mathrm{NaN}_{3}$ to $35.12 \mathrm{~cm}$ at $0 \%$. NERICA L34 ranged from $27.25 \mathrm{~cm}$ at $0.032 \% \mathrm{NaN}_{3}$ to $34.12 \mathrm{~cm}$ in control. NERICA L-47 ranged from $15.00 \mathrm{~cm}$ at $0.016 \%$ $\mathrm{NaN}_{3}$ to $31.00 \mathrm{~cm}$ in control.

At 5 WAP, FARO- 44 was $54.38 \mathrm{~cm}, 54.88 \mathrm{~cm}$, and $59.00 \mathrm{~cm}$ at control, $0 \% \mathrm{NaN}_{3}$, and $0.008 \% \mathrm{NaN}_{3}$, respectively. FARO-52 was $56.75 \mathrm{~cm}, 59.75 \mathrm{~cm}$, and 63.62 $\mathrm{cm}$ at control, $0 \% \mathrm{NaN}_{3}$, and $0.008 \% \mathrm{NaN}_{3}$, respectively.
FARO-57 was $61.50 \mathrm{~cm}, 63.75 \mathrm{~cm}$, and $61.75 \mathrm{~cm}$ at control, $0 \% \mathrm{NaN}_{3}$, and $0.004 \% \mathrm{NaN}_{3}$, respectively. NERICA L-34 had control of $56.25 \mathrm{~cm}, 0 \% \mathrm{NaN}_{3}$ of $54.62 \mathrm{~cm}$, and $0.008 \% \mathrm{NaN}_{3}$, and $0.032 \% \mathrm{NaN}_{3}$ of $54.75 \mathrm{~cm}$ each. NERICA L-47 was $58.50 \mathrm{~cm}$ for control, $56.62 \mathrm{~cm}$ for $0 \%$ $\mathrm{NaN}_{3}$, and $54.38 \mathrm{~cm}$ for $0.004 \% \mathrm{NaN}_{3}$.

At 11 WAP, FARO-44 was $78.12 \mathrm{~cm}, 70.38 \mathrm{~cm}$, and $78.28 \mathrm{~cm}$ in control, $0 \% \mathrm{NaN}_{3}$, and $0.008 \% \mathrm{NaN}_{3}$, respectively. FARO- 52 was $86.55 \mathrm{~cm}, 88.75 \mathrm{~cm}$, and 85.67 $\mathrm{cm}$ for control, $0 \% \mathrm{NaN}_{3}$, and $0.008 \% \mathrm{NaN}_{3}$, respectively. FARO-57 was $97.12 \mathrm{~cm}, 95.25 \mathrm{~cm}$, and $97.10 \mathrm{~cm}$ for control, $0 \% \mathrm{NaN}_{3}$, and $0.004 \% \mathrm{NaN}_{3}$, respectively. NERICA L-34 was control of $82.42 \mathrm{~cm}, 0 \% \mathrm{NaN}_{3}$ of 
Table 3 Plant heights of the five rice varieties (CM)

\begin{tabular}{|c|c|c|c|c|c|c|c|}
\hline \multirow[t]{2}{*}{ Variety } & \multirow{2}{*}{$\begin{array}{l}\text { NaN3 } \\
\text { treatment } \\
\text { level }\end{array}$} & \multicolumn{6}{|c|}{ Plant heights $(\mathrm{cm})$} \\
\hline & & Week 3 & Week 4 & Week 5 & Week 7 & Week 9 & Week 11 \\
\hline FARO-44 & Control & $36.00 \pm 1.78^{d}$ & $48.12 \pm 1.86^{b}$ & $54.38 \pm 2.97^{b}$ & $55.12 \pm 6.46^{\mathrm{ab}}$ & $64.38 \pm 5.29^{a}$ & $78.12 \pm 4.92^{\mathrm{ab}}$ \\
\hline FARO-44 & $0 \%$ & $33.30 \pm 1.21^{\mathrm{bcd}}$ & $45.75 \pm 1.65^{b}$ & $54.88 \pm 5.78^{b}$ & $54.75 \pm 5.84^{\mathrm{ab}}$ & $58.00 \pm 6.18^{a}$ & $70.38 \pm 6.52^{\mathrm{a}}$ \\
\hline FARO-44 & $0.004 \%$ & $33.50 \pm 0.96^{\mathrm{bcd}}$ & $43.00 \pm 3.24^{b}$ & $50.75 \pm 3.20^{b}$ & $56.75 \pm 2.02^{\mathrm{ab}}$ & $60.50 \pm 2.60^{a}$ & $74.62 \pm 5.85^{\mathrm{ab}}$ \\
\hline FARO-44 & $0.008 \%$ & $33.25 \pm 1.32^{\mathrm{bcd}}$ & $45.25 \pm 2.93^{b}$ & $59.00 \pm 1.47^{b}$ & $64.75 \pm 1.32^{\mathrm{ab}}$ & $66.25 \pm 1.18^{a}$ & $78.28 \pm 1.46^{\mathrm{ab}}$ \\
\hline FARO-44 & $0.016 \%$ & $33.38 \pm 1.38^{\mathrm{bcd}}$ & $44.75 \pm 2.72^{b}$ & $53.20 \pm 2.95^{b}$ & $61.50 \pm 0.65^{a b}$ & $65.25 \pm 0.85^{a}$ & $75.40 \pm 2.24^{\mathrm{ab}}$ \\
\hline FARO-44 & $0.032 \%$ & $32.38 \pm 1.07^{\mathrm{bcd}}$ & $43.50 \pm 3.12^{b}$ & $53.25 \pm 3.15^{b}$ & $58.50 \pm 3.80^{a b}$ & $63.75 \pm 3.43^{\mathrm{a}}$ & $74.85 \pm 3.48^{\mathrm{ab}}$ \\
\hline FARO-52 & Control & $35.35 \pm 1.49^{\mathrm{cd}}$ & $46.00 \pm 3.81^{b}$ & $56.75 \pm 1.59^{b}$ & $63.50 \pm 0.87^{a b}$ & $71.00 \pm 2.61^{\mathrm{a}}$ & $86.55 \pm 5.25^{\mathrm{ab}}$ \\
\hline FARO-52 & $0 \%$ & $33.75 \pm 1.65^{\mathrm{bcd}}$ & $46.50 \pm 3.23^{b}$ & $59.62 \pm 2.81^{b}$ & $66.50 \pm 2.26^{\mathrm{ab}}$ & $75.25 \pm 1.93^{\mathrm{a}}$ & $88.75 \pm 3.93^{\mathrm{ab}}$ \\
\hline FARO-52 & $0.004 \%$ & $30.50 \pm 3.38^{\mathrm{bcd}}$ & $45.00 \pm 6.98^{b}$ & $51.50 \pm 9.97^{\mathrm{b}}$ & $55.50 \pm 11.29^{\mathrm{ab}}$ & $61.25 \pm 12.87^{\mathrm{a}}$ & $82.97 \pm 5.74^{\mathrm{ab}}$ \\
\hline FARO-52 & $0.008 \%$ & $34.50 \pm 1.94^{\mathrm{cd}}$ & $53.00 \pm 3.49^{b}$ & $63.62 \pm 3.21^{b}$ & $69.75 \pm 1.60^{b}$ & $72.50 \pm 1.50^{\mathrm{a}}$ & $85.67 \pm 2.38^{\mathrm{ab}}$ \\
\hline FARO-52 & $0.016 \%$ & $33.25 \pm 0.85^{\mathrm{bcd}}$ & $46.00 \pm 1.63^{b}$ & $57.00 \pm 2.04^{b}$ & $67.00 \pm 2.48^{\mathrm{ab}}$ & $71.25 \pm 2.75^{\mathrm{a}}$ & $82.35 \pm 5.74^{\mathrm{ab}}$ \\
\hline FARO-52 & $0.032 \%$ & $29.52 \pm 0.87^{b c d}$ & $43.62 \pm 2.59^{b}$ & $57.75 \pm 2.18^{b}$ & $65.25 \pm 2.32^{\mathrm{ab}}$ & $71.25 \pm 3.30^{a}$ & $83.90 \pm 3.56^{\mathrm{ab}}$ \\
\hline FARO-57 & Control & $34.75 \pm 1.84^{\mathrm{cd}}$ & $52.00 \pm 1.23^{b}$ & $61.50 \pm 1.04^{b}$ & $67.00 \pm 3.08^{\mathrm{ab}}$ & $74.00 \pm 4.51^{\mathrm{a}}$ & $97.12 \pm 5.35^{b}$ \\
\hline FARO-57 & $0 \%$ & $35.12 \pm 1.66^{\mathrm{cd}}$ & $54.50 \pm 3.12^{b}$ & $63.75 \pm 1.32^{b}$ & $69.00 \pm 2.66^{b}$ & $73.00 \pm 3.34^{a}$ & $95.25 \pm 8.23^{b}$ \\
\hline FARO-57 & $0.004 \%$ & $34.25 \pm 1.93^{\mathrm{bcd}}$ & $49.00 \pm 1.63^{b}$ & $61.75 \pm 1.65^{b}$ & $68.00 \pm 1.47^{\mathrm{ab}}$ & $75.50 \pm 1.56^{a}$ & $97.10 \pm 4.73^{b}$ \\
\hline FARO-57 & $0.008 \%$ & $33.00 \pm 2.04^{\mathrm{bcd}}$ & $48.50 \pm 3.50^{b}$ & $58.75 \pm 3.20^{b}$ & $64.25 \pm 1.89^{\mathrm{ab}}$ & $71.00 \pm 1.35^{\mathrm{a}}$ & $96.40 \pm 5.31^{b}$ \\
\hline FARO-57 & $0.016 \%$ & $30.25 \pm 1.70^{\mathrm{bcd}}$ & $46.75 \pm 3.97^{b}$ & $60.75 \pm 3.82^{b}$ & $69.25 \pm 2.66^{b}$ & $74.75 \pm 3.52^{a}$ & $88.20 \pm 5.14^{\mathrm{ab}}$ \\
\hline FARO-57 & $0.032 \%$ & $30.62 \pm 2.79^{b c d}$ & $44.00 \pm 2.12^{b}$ & $56.75 \pm 1.25^{b}$ & $67.00 \pm 1.68^{\mathrm{ab}}$ & $71.75 \pm 1.32^{\mathrm{a}}$ & $90.22 \pm 4.03^{a b}$ \\
\hline NERICA L-34 & Control & $34.12 \pm 1.23^{\mathrm{bcd}}$ & $45.60 \pm 0.69^{b}$ & $56.25 \pm 2.25^{b}$ & $62.75 \pm 2.46^{\mathrm{ab}}$ & $70.75 \pm 1.65^{a}$ & $82.42 \pm 3.48^{\mathrm{ab}}$ \\
\hline NERICA L-34 & $0 \%$ & $32.25 \pm 2.53^{\mathrm{bcd}}$ & $48.25 \pm 0.68^{b}$ & $54.62 \pm 2.14^{b}$ & $59.25 \pm 3.04^{\mathrm{ab}}$ & $67.00 \pm 2.83^{a}$ & $85.53 \pm 6.61^{a b}$ \\
\hline NERICA L-34 & $0.004 \%$ & $31.88 \pm 1.23^{\mathrm{bcd}}$ & $41.50 \pm 3.66^{b}$ & $54.62 \pm 3.25^{b}$ & $59.75 \pm 2.32^{\mathrm{ab}}$ & $65.50 \pm 2.83^{a}$ & $79.88 \pm 2.97^{\mathrm{ab}}$ \\
\hline NERICA L-34 & $0.008 \%$ & $31.75 \pm 0.95^{\mathrm{bcd}}$ & $45.50 \pm 2.87^{b}$ & $54.75 \pm 1.32^{b}$ & $61.50 \pm 2.63^{a b}$ & $65.88 \pm 1.74^{\mathrm{a}}$ & $77.40 \pm 5.44^{\mathrm{ab}}$ \\
\hline NERICA L-34 & $0.016 \%$ & $31.00 \pm 0.23^{\mathrm{bcd}}$ & $47.25 \pm 1.80^{b}$ & $54.25 \pm 2.66^{b}$ & $61.75 \pm 3.59^{a b}$ & $67.75 \pm 2.46^{\mathrm{a}}$ & $80.88 \pm 6.30^{\mathrm{ab}}$ \\
\hline NERICA L-34 & $0.032 \%$ & $27.25 \pm 2.21^{b c}$ & $42.00 \pm 3.56^{b}$ & $54.75 \pm 3.50^{b}$ & $62.00 \pm 2.61^{a b}$ & $69.75 \pm 1.55^{\mathrm{a}}$ & $80.85 \pm 4.61^{\mathrm{ab}}$ \\
\hline NERICA L-47 & Control & $31.00 \pm 1.78^{\mathrm{bcd}}$ & $44.25 \pm 1.70^{b}$ & $58.50 \pm 1.66^{b}$ & $63.25 \pm 2.63^{\mathrm{ab}}$ & $67.50 \pm 4.48^{\mathrm{a}}$ & $81.70 \pm 3.79^{\mathrm{ab}}$ \\
\hline NERICA L-47 & $0 \%$ & $27.75 \pm 2.66^{b c d}$ & $46.00 \pm 3.81^{b}$ & $56.62 \pm 1.28^{b}$ & $62.50 \pm 1.19^{\mathrm{ab}}$ & $69.25 \pm 1.93^{\mathrm{a}}$ & $79.50 \pm 3.31^{\mathrm{ab}}$ \\
\hline NERICA L-47 & $0.004 \%$ & $26.25 \pm 0.63^{b}$ & $41.00 \pm 1.47^{b}$ & $54.38 \pm 2.48^{b}$ & $64.25 \pm 3.07^{\mathrm{ab}}$ & $71.00 \pm 4.49^{a}$ & $93.12 \pm 5.87^{a b}$ \\
\hline NERICA L-47 & $0.008 \%$ & $16.00 \pm 0.58^{a}$ & $32.33 \pm 2.03^{a}$ & $50.00 \pm 1.73^{a}$ & $56.83 \pm 4.51^{\mathrm{ab}}$ & $65.67 \pm 3.18^{a}$ & $83.77 \pm 4.56^{\mathrm{ab}}$ \\
\hline NERICA L-47 & $0.016 \%$ & $15.00 \pm 0^{a}$ & $48.00 \pm 0^{b}$ & $36.00 \pm 0^{b}$ & $50.00 \pm 0^{a}$ & $64.00 \pm 0^{a}$ & $84.80 \pm 0^{\mathrm{ab}}$ \\
\hline
\end{tabular}

Mean \pm standard error. Mean of four replicates. NERICA L-47 (0.008 \%); mean of three replicates. NERICA L-47 (0.016 \%); no replicate. Different alphabets in columns indicate significant differences at $5 \%$ level of probability

$85.53 \mathrm{~cm}$, and $80.88 \mathrm{~cm}$ for $0.016 \% \mathrm{NaN}_{3}$. NERICA L-47 was $81.70 \mathrm{~cm}, 79.50 \mathrm{~cm}$, and $93.12 \mathrm{~cm}$ for control, $0 \%$ $\mathrm{NaN}_{3}$, and $0.004 \% \mathrm{NaN}_{3}$, respectively.

Table 4 shows the chlorophyll content indexes (CCI) of the five rice varieties. Across varieties at 5 weeks after planting (5 WAP), $0.008 \% \mathrm{NaN}_{3}$ of FARO-52 had the highest CCI of 8.83 while NERICA L-47 had the lowest CCI of 1.60. At 12 WAP, control of FARO-44 had the highest CCI of 15.40 , and the lowest value of CCI was 11.20 for FARO-52 at $0.016 \% \mathrm{NaN}_{3}$.

Table 5 shows the earliness to panicle emergence of the five varieties. The NERICAs had the earliest panicle emergence at 81 DAP. However, among the NERICAs, NERICA L-47, 0.016\% $\mathrm{NaN}_{3}$ was the latest at 91 DAP.
Next was FARO-44 with the earliest at 85 DAP and FARO-57 with the earliest at 89 DAP. Control of FARO57 was at 97 DAP. FARO-52 was the latest of all the varieties with the earliest panicle emerging at 106 DAP.

Table 6 shows percentage lodging in the five rice varieties. Control of FARO- 44 had the highest value of $50 \%$ lodging and $0.032 \% \mathrm{NaN}_{3}$ of $12.50 \%$. However, there was no lodging at $0 \% \mathrm{NaN}_{3}$. There was no lodging in all the treatment levels, control inclusive in FARO-52. FARO-57 had highest values at $0.004 \% \mathrm{NaN}_{3}$ and $0.016 \% \mathrm{NaN}_{3}$ with $100 \%$ lodging and lowest at $0.032 \%$ $\mathrm{NaN}_{3}$ with 50\%. For NERICA L-34, percentage lodging was $12.50 \%$ each in control and $0 \% \mathrm{NaN}_{3}$. There was no lodging at other treatment levels. NERICA L-47 had 
Table 4 Chlorophyll content index (CCI) of the five rice varieties at 5 WAP and 12 WAP

\begin{tabular}{|c|c|c|c|}
\hline \multirow[t]{2}{*}{ Variety } & \multirow{2}{*}{$\begin{array}{l}\text { NaN3 } \\
\text { treatment } \\
\text { level }\end{array}$} & \multicolumn{2}{|l|}{$\mathrm{CCl}$} \\
\hline & & 5 WAP & 12 WAP \\
\hline FARO-44 & Control & $4.35 \pm 0.78^{b}$ & $15.40 \pm 2.01^{\mathrm{a}}$ \\
\hline FARO-44 & $0 \%$ & $4.48 \pm 0.93^{\mathrm{ab}}$ & $13.00 \pm 1.39^{a}$ \\
\hline FARO-44 & $0.004 \%$ & $5.15 \pm 0.90^{\mathrm{abc}}$ & $11.83 \pm 1.41^{a}$ \\
\hline FARO-44 & $0.008 \%$ & $5.68 \pm 0.41^{b c}$ & $12.22 \pm 1.16^{\mathrm{a}}$ \\
\hline FARO-44 & $0.016 \%$ & $5.03 \pm 1.06^{\mathrm{abc}}$ & $13.57 \pm 0.61^{a}$ \\
\hline FARO-44 & $0.032 \%$ & $4.88 \pm 0.98^{\mathrm{abc}}$ & $12.12 \pm 1.51^{\mathrm{a}}$ \\
\hline FARO-52 & Control & $6.23 \pm 1.38^{b c}$ & $11.25 \pm 1.20^{\mathrm{a}}$ \\
\hline FARO-52 & $0 \%$ & $6.75 \pm 1.49^{b c}$ & $14.20 \pm 1.24^{a}$ \\
\hline FARO-52 & $0.004 \%$ & $6.00 \pm 1.76^{b c}$ & $12.33 \pm 2.33^{\mathrm{a}}$ \\
\hline FARO-52 & $0.008 \%$ & $8.83 \pm 0.51^{c}$ & $11.95 \pm 0.95^{\mathrm{a}}$ \\
\hline FARO-52 & $0.016 \%$ & $5.98 \pm 0.33^{b c}$ & $11.20 \pm 0.93^{\mathrm{a}}$ \\
\hline FARO-52 & $0.032 \%$ & $7.48 \pm 0.98^{b c}$ & $13.58 \pm 1.19^{\mathrm{a}}$ \\
\hline FARO-57 & Control & $7.55 \pm 0.90^{b c}$ & $14.43 \pm 0.80^{\mathrm{a}}$ \\
\hline FARO-57 & $0 \%$ & $8.28 \pm 6.58^{b c}$ & $13.52 \pm 0.81^{\mathrm{a}}$ \\
\hline FARO-57 & $0.004 \%$ & $6.58 \pm 0.82^{b c}$ & $14.07 \pm 0.59^{\mathrm{a}}$ \\
\hline FARO-57 & $0.008 \%$ & $6.70 \pm 0.94^{b c}$ & $14.23 \pm 1.11^{\mathrm{a}}$ \\
\hline FARO-57 & $0.016 \%$ & $5.59 \pm 1.09^{b c}$ & $12.70 \pm 0.42^{\mathrm{a}}$ \\
\hline FARO-57 & $0.032 \%$ & $4.55 \pm 0.83^{\mathrm{ab}}$ & $13.85 \pm 1.51^{\mathrm{a}}$ \\
\hline NERICA L-34 & Control & $6.85 \pm 0.94^{b c}$ & $16.40 \pm 1.27^{\mathrm{a}}$ \\
\hline NERICA L-34 & $0 \%$ & $6.30 \pm 0.72^{b c}$ & $13.22 \pm 1.31^{\mathrm{a}}$ \\
\hline NERICA L-34 & $0.004 \%$ & $4.98 \pm 0.86^{\mathrm{abc}}$ & $12.75 \pm 0.92^{\mathrm{a}}$ \\
\hline NERICA L-34 & $0.008 \%$ & $5.76 \pm 0.56^{b c}$ & $12.00 \pm 2.57^{\mathrm{a}}$ \\
\hline NERICA L-34 & $0.016 \%$ & $5.93 \pm 1.15^{\mathrm{bc}}$ & $14.00 \pm 1.95^{\mathrm{a}}$ \\
\hline NERICA L-34 & $0.032 \%$ & $6.53 \pm 0.36^{b c}$ & $14.72 \pm 1.70^{a}$ \\
\hline NERICA L-47 & Control & $5.88 \pm 0.87^{b c}$ & $12.12 \pm 1.28^{\mathrm{a}}$ \\
\hline NERICA L-47 & $0 \%$ & $7.03 \pm 0.63^{b c}$ & $13.88 \pm 2.25^{\mathrm{a}}$ \\
\hline NERICA L-47 & $0.004 \%$ & $5.33 \pm 0.20^{b c}$ & $13.18 \pm 1.68^{\mathrm{a}}$ \\
\hline NERICA L-47 & $0.008 \%$ & $2.27 \pm 0.34^{a}$ & $12.63 \pm 0.93^{\mathrm{a}}$ \\
\hline NERICA L-47 & $0.016 \%$ & $1.60 \pm 0^{\mathrm{a}}$ & $12.60 \pm 0^{a}$ \\
\hline
\end{tabular}

Mean \pm standard error. Mean of four replicates. NERICA L-47 (0.008 \%); mean of three replicates. NERICA L-47 (0.016 \%); no replicate. Different alphabets in columns indicate significant differences at $5 \%$ level of probability

highest lodging at $0 \% \mathrm{NaN}_{3}$ with $37.50 \%$ and $25 \%$ in control. There was no lodging in $0.004 \% \mathrm{NaN}_{3}, 0.008 \%$ $\mathrm{NaN}_{3}$, and $0.016 \% \mathrm{NaN}_{3}$. The overall varietal lodging shows FARO-57 as the most lodged with $81.25 \%$ and no lodging in FARO-52.

Table 7 shows the yield parameters of the five rice varieties. For number of tillers per plant at 18 WAP, FARO-44 had highest number at control with 6 tillers per plant. It had 4 tillers per plant at $0 \% \mathrm{NaN}_{3}$ and 6 tillers per plant at $0.032 \% \mathrm{NaN}_{3}$. FARO-52 had 10 tillers per plant at control, 8 tillers per plant at $0 \% \mathrm{NaN}_{3}$, and 10 tillers per plant at $0.032 \% \mathrm{NaN}_{3}$. FARO-57 had 5 tillers per plant at control, 4 tillers per plant at $0 \%$ $\mathrm{NaN}_{3}$, and 5 tillers per plant at $0.004 \% \mathrm{NaN}_{3}$ and $0.008 \% \mathrm{NaN}_{3}$. NERICA L-34 had 8 tillers per plant each at control and level 0 with 9 tillers per plant $0.032 \%$ $\mathrm{NaN}_{3}$. NERICA L-47 had 7 tillers per plant for control, 9 tillers per plant for $0 \% \mathrm{NaN}_{3}$, and between 10 and 12 tillers per plant in the other levels.

For number of panicles per plant, control of FARO-44 had 7 panicles per plant, 4 panicles per plant in $0 \%$ $\mathrm{NaN}_{3}$, and 7 panicles per plant in $0.004 \% \mathrm{NaN}_{3}$ and $0.032 \% \mathrm{NaN}_{3}$. FARO-52 had 8 panicles per plant in control, 7 panicles per plant in $0 \% \mathrm{NaN}_{3}$, and 8 panicles per plant in $0.032 \% \mathrm{NaN}_{3}$. FARO-57 had control of 4 panicles per plant, $0 \% \mathrm{NaN}_{3}$ of 4 panicles per plant, and $0.032 \% \mathrm{NaN}_{3}$ of 9 panicles per plant. NERICA L-34 had control of 8 panicles per plant, $0 \% \mathrm{NaN}_{3}$ of 4 panicles per plant, and $0.032 \% \mathrm{NaN}_{3}$ of 9 panicles per plant. NERICA L-47 had control of 10 panicles per plant, $0 \%$ $\mathrm{NaN}_{3}$ of 7 panicles per plant, and $0.016 \% \mathrm{NaN}_{3}$ of 17 panicles per plant.

For the control plants, 100-grain weight for FARO-44 was $3.02 \mathrm{~g}, 2.93 \mathrm{~g}$ in $0 \% \mathrm{NaN}_{3}$, and $3.03 \mathrm{~g}$ in $0.004 \%$ $\mathrm{NaN}_{3}$. FARO-52 was $2.47 \mathrm{~g}$ in control, $2.59 \mathrm{~g}$ in $0 \%$ $\mathrm{NaN}_{3}$, and $2.59 \mathrm{~g}$ at $0.004 \% \mathrm{NaN}_{3}$. FARO-57 was $3.02 \mathrm{~g}$ in control, $3.12 \mathrm{~g}$ in $0 \%$, and $3.06 \mathrm{~g}$ in $0.004 \%$. NERICA L-34 was control of $2.57 \mathrm{~g}, 0 \% \mathrm{NaN}_{3}$ of $2.45 \mathrm{~g}$, and $2.68 \mathrm{~g}$ in $0.016 \% \mathrm{NaN}_{3}$. NERICA L-47 had control of $2.91 \mathrm{~g}, 0 \%$ $\mathrm{NaN}_{3}$ of $2.19 \mathrm{~g}$, and $0.008 \% \mathrm{NaN}_{3}$ of $3.01 \mathrm{~g}$.

Biomass for FARO-44 was $8.15 \mathrm{~g}$ for control, $4.98 \mathrm{~g}$ for $0 \% \mathrm{NaN}_{3}$, and $6.13 \mathrm{~g}$ for $0.016 \% \mathrm{NaN}_{3}$. FARO-52 had biomass of $15.01 \mathrm{~g}$ for control, $15.08 \mathrm{~g}$ for $0 \% \mathrm{NaN}_{3}$, and $18.02 \mathrm{~g}$ for $0.032 \% \mathrm{NaN}_{3}$. FARO-57 was $9.37 \mathrm{~g}$ for control, $8.59 \mathrm{~g}$ for $0 \% \mathrm{NaN}_{3}$, and $11.14 \mathrm{~g}$ for $0.004 \% \mathrm{NaN}_{3}$.

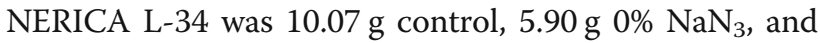
$12.09 \mathrm{~g}$ for $0.032 \% \mathrm{NaN}_{3}$. Biomass for NERICA L-47 was $8.61 \mathrm{~g}, 11.56 \mathrm{~g}$, and $19.20 \mathrm{~g}$ for control, $0 \% \mathrm{NaN}_{3}$, and $0.004 \% \mathrm{NaN}_{3}$, respectively.

Table 8 shows the estimate of parameters of variability, heritability, and genetic gain of some characters of the rice varieties in the $M_{1}$ generation. Characters estimated for were percentage emergence, number of tillers per plant, number of panicles per plant, 100-grain weight, and percentage lodging. Heritability for percentage emergence, number of tillers per plant, number of panicles per plant, 100-grain weight, and percentage lodging were $46.82 \%, 78.06 \%, 78.74 \%, 98.15 \%$, and $25.60 \%$, respectively.

Table 9 shows the effect of sodium azide on some parameters comparatively between $M_{1}$ and $M_{2}$ of selected progenies. Parameters studied were percentage emergence, plant heights at $7 \mathrm{WAP}$, and chlorophyll mutations. Percentage emergence across in $M_{1}$ ranged between 1.25 and $98.75 \% . \mathrm{M}_{2}$ ranged between 5 and $100 \%$. Plant heights in $M_{1}$ ranged between 50.00 and 
Table 5 Number of days to flowering and panicle emergence in the five rice varieties

\begin{tabular}{|c|c|c|c|c|c|c|c|c|c|c|c|c|c|c|c|c|}
\hline \multirow[t]{2}{*}{ Variety } & \multirow{2}{*}{$\begin{array}{l}\text { NaN3 } \\
\text { treatment } \\
\text { level }\end{array}$} & \multicolumn{15}{|c|}{ Earliness to panicle emergence } \\
\hline & & $\begin{array}{l}81 \\
\text { DAP }\end{array}$ & $\begin{array}{l}82 \\
\text { DAP }\end{array}$ & $\begin{array}{l}84 \\
\text { DAP }\end{array}$ & $\begin{array}{l}85 \\
\text { DAP }\end{array}$ & $\begin{array}{l}88 \\
\text { DAP }\end{array}$ & $\begin{array}{l}89 \\
\text { DAP }\end{array}$ & $\begin{array}{l}91 \\
\text { DAP }\end{array}$ & $\begin{array}{l}95 \\
\text { DAP }\end{array}$ & $\begin{array}{l}97 \\
\text { DAP }\end{array}$ & $\begin{array}{l}99 \\
\text { DAP }\end{array}$ & $\begin{array}{l}106 \\
\text { DAP }\end{array}$ & $\begin{array}{l}110 \\
\text { DAP }\end{array}$ & $\begin{array}{l}118 \\
\text { DAP }\end{array}$ & $\begin{array}{l}120 \\
\text { DAP }\end{array}$ & $\begin{array}{l}\text { Total no. of } \\
\text { plants }\end{array}$ \\
\hline FARO-44 & Control & - & - & - & 1 & 4 & 4 & 8 & 9 & 10 & 11 & 14 & 16 & 16 & 17 & 4 \\
\hline FARO-44 & $0 \%$ & - & - & - & 2 & 4 & 4 & 5 & 6 & 6 & 7 & 9 & 11 & 12 & 12 & 4 \\
\hline FARO-44 & $0.004 \%$ & - & - & - & - & 2 & 3 & 4 & 6 & 6 & 7 & 9 & 13 & 14 & 15 & 4 \\
\hline FARO-44 & $0.008 \%$ & - & - & - & - & 4 & 4 & 5 & 8 & 8 & 8 & 10 & 13 & 15 & 15 & 4 \\
\hline FARO-44 & $0.016 \%$ & - & - & - & - & 2 & 2 & 5 & 7 & 8 & 9 & 11 & 14 & 14 & 14 & 4 \\
\hline FARO-44 & $0.032 \%$ & - & - & - & - & 2 & 2 & 3 & 3 & 7 & 8 & 13 & 15 & 16 & 18 & 4 \\
\hline FARO-52 & Control & - & - & - & - & - & - & - & - & - & - & 1 & 5 & 8 & 11 & 4 \\
\hline FARO-52 & $0 \%$ & - & - & - & - & - & - & - & - & - & - & 1 & 4 & 7 & 9 & 4 \\
\hline FARO-52 & $0.004 \%$ & - & - & - & - & - & - & - & - & - & - & - & 1 & 7 & 10 & 4 \\
\hline FARO-52 & $0.008 \%$ & - & - & - & - & - & - & - & - & - & - & 2 & 7 & 11 & 15 & 4 \\
\hline FARO-52 & $0.016 \%$ & - & - & - & - & - & - & - & - & - & - & 1 & 2 & 8 & 10 & 4 \\
\hline FARO-52 & $0.032 \%$ & - & - & - & - & - & - & - & - & - & - & - & 1 & 6 & 8 & 4 \\
\hline FARO-57 & Control & - & - & - & - & - & - & - & - & - & 1 & 7 & 9 & 11 & 13 & 4 \\
\hline FARO-57 & $0 \%$ & - & - & - & - & - & - & - & 2 & 2 & 4 & 8 & 9 & 9 & 10 & 4 \\
\hline FARO-57 & $0.004 \%$ & - & - & - & - & - & - & - & 1 & 2 & 5 & 7 & 8 & 10 & 12 & 4 \\
\hline FARO-57 & $0.008 \%$ & - & - & - & - & - & - & 1 & 2 & 3 & 3 & 6 & 8 & 8 & 9 & 4 \\
\hline FARO-57 & $0.016 \%$ & - & - & - & - & - & - & - & 1 & 2 & 3 & 6 & 8 & 9 & 10 & 4 \\
\hline FARO-57 & $0.032 \%$ & - & - & - & - & - & 1 & 1 & 2 & 2 & 3 & 4 & 8 & 10 & 11 & 4 \\
\hline $\begin{array}{l}\text { NERICA L- } \\
34\end{array}$ & Control & 1 & 1 & 4 & 6 & 9 & 9 & 11 & 11 & 12 & 12 & 15 & 15 & 20 & 22 & 4 \\
\hline $\begin{array}{l}\text { NERICA L- } \\
34\end{array}$ & $0 \%$ & 2 & 4 & 6 & 6 & 7 & 8 & 9 & 9 & 9 & 9 & 10 & 11 & 12 & 12 & 4 \\
\hline $\begin{array}{l}\text { NERICA L- } \\
34\end{array}$ & $0.004 \%$ & 2 & 3 & 3 & 5 & 9 & 9 & 11 & 11 & 11 & 11 & 12 & 14 & 15 & 17 & 4 \\
\hline $\begin{array}{l}\text { NERICA L- } \\
34\end{array}$ & $0.008 \%$ & - & - & 2 & 4 & 7 & 8 & 9 & 9 & 10 & 10 & 12 & 14 & 16 & 16 & 4 \\
\hline $\begin{array}{l}\text { NERICA L- } \\
34\end{array}$ & $0.016 \%$ & 1 & 2 & 5 & 6 & 10 & 10 & 12 & 12 & 12 & 12 & 13 & 17 & 23 & 25 & 4 \\
\hline $\begin{array}{l}\text { NERICA L- } \\
34\end{array}$ & $0.032 \%$ & 2 & 2 & 3 & 3 & 4 & 4 & 5 & 6 & 9 & 12 & 16 & 18 & 25 & 26 & 4 \\
\hline $\begin{array}{l}\text { NERICA L- } \\
47\end{array}$ & Control & 2 & 2 & 3 & 3 & 4 & 5 & 6 & 7 & 12 & 12 & 16 & 25 & 28 & 26 & 4 \\
\hline $\begin{array}{l}\text { NERICA L- } \\
47\end{array}$ & $0 \%$ & 1 & 1 & 5 & 6 & 8 & 9 & 12 & 14 & 16 & 18 & 21 & 21 & 25 & 25 & 4 \\
\hline $\begin{array}{l}\text { NERICA L- } \\
47\end{array}$ & $0.004 \%$ & - & - & 4 & 8 & 9 & 8 & 11 & 15 & 16 & 17 & 26 & 26 & 29 & 32 & 4 \\
\hline $\begin{array}{l}\text { NERICA L- } \\
47\end{array}$ & $0.008 \%$ & - & - & - & - & - & - & - & 1 & 6 & 9 & 16 & 19 & 20 & 21 & 3 \\
\hline $\begin{array}{l}\text { NERICA L- } \\
47\end{array}$ & $0.016 \%$ & - & - & - & 1 & 2 & 3 & 4 & 4 & 4 & 4 & 4 & 6 & 10 & 10 & 1 \\
\hline
\end{tabular}

$68.00 \mathrm{~cm}$, with $12 \mathrm{~cm}$ and $67.75 \mathrm{~cm}$ in $\mathrm{M}_{2}$. Chlorophyll mutations observed in the present studies were striata, albino, and viridis with the striata form being the most predominant.

Figures 1, 2, and 3 show the RAPD gel electrophoresis of nineteen selected $M_{1}$ progenies of control and mutant plants using RAPD primer OPR 02 (5'-CAC AGC TGC
C-3'), OPC 04 (5' -CCG CAT CTA C-3'), and OPC 05 (5'-GAT GAC CGC C-3'), respectively. The RAPD marker presented in Fig. 1 reveals variation among salinity-exposed rice cultivars after treatment with sodium azide. FARO-44 (control), FARO-52 (control), NERICA L-47 (control), FARO-52 (0.008 \%), FARO-52 (0.016\%), FARO-57 (0\%), FARO-57 (0.008\%), NERICA 
Table 6 Percentage lodging of the five rice varieties

\begin{tabular}{|c|c|c|c|}
\hline Variety & NaN3 treatment level & Percentage lodging & Varietal percentage lodging \\
\hline FARO-44 & Control & $50.00 \pm 20.41^{\mathrm{abcd}}$ & $27.08 \pm 7.51^{b}$ \\
\hline FARO-44 & $0 \%$ & $0.00^{\mathrm{a}}$ & \\
\hline FARO-44 & $0.004 \%$ & $37.50 \pm 12.50^{\mathrm{abc}}$ & \\
\hline FARO-44 & $0.008 \%$ & $37.50 \pm 23.94^{\mathrm{abc}}$ & \\
\hline FARO-44 & $0.016 \%$ & $37.50 \pm 12.50^{\mathrm{abc}}$ & \\
\hline FARO-44 & $0.032 \%$ & $12.50 \pm 12.50^{a}$ & \\
\hline FARO-52 & Control & $0.00^{\mathrm{a}}$ & $0.00^{\mathrm{a}}$ \\
\hline FARO-52 & $0 \%$ & $0.00^{\mathrm{a}}$ & \\
\hline FARO-52 & $0.004 \%$ & $0.00^{\mathrm{a}}$ & \\
\hline FARO-52 & $0.008 \%$ & $0.00^{\mathrm{a}}$ & \\
\hline FARO-52 & $0.016 \%$ & $0.00^{\mathrm{a}}$ & \\
\hline FARO-52 & $0.032 \%$ & $0.00^{\mathrm{a}}$ & \\
\hline FARO-57 & Control & $75.00 \pm 25.00^{\mathrm{bcd}}$ & $81.25 \pm 7.74^{b}$ \\
\hline FARO-57 & $0 \%$ & $87.50 \pm 12.50^{c d}$ & \\
\hline FARO-57 & $0.004 \%$ & $100.00 \pm 0^{c d}$ & \\
\hline FARO-57 & $0.008 \%$ & $75.00 \pm 25.00^{\mathrm{bcd}}$ & \\
\hline FARO-57 & $0.016 \%$ & $100.00 \pm 0^{d}$ & \\
\hline FARO-57 & $0.032 \%$ & $50.00 \pm 20.41^{\mathrm{abcd}}$ & \\
\hline NERICA L-34 & Control & $12.50 \pm 12.50^{a}$ & $4.17 \pm 2.64^{\mathrm{a}}$ \\
\hline NERICA L-34 & $0 \%$ & $12.50 \pm 12.50^{\mathrm{a}}$ & \\
\hline NERICA L-34 & $0.004 \%$ & $0.00^{\mathrm{a}}$ & \\
\hline NERICA L-34 & $0.008 \%$ & $0.00^{\mathrm{a}}$ & \\
\hline NERICA L-34 & $0.016 \%$ & $0.00^{\mathrm{a}}$ & \\
\hline NERICA L-34 & $0.032 \%$ & $0.00^{\mathrm{a}}$ & \\
\hline NERICA L-47 & Control & $25.00 \pm 14.43^{\mathrm{ab}}$ & $12.50 \pm 7.91^{\mathrm{a}}$ \\
\hline NERICA L-47 & $0 \%$ & $37.50 \pm 23.94^{\mathrm{abc}}$ & \\
\hline NERICA L-47 & $0.004 \%$ & $0.00^{\mathrm{a}}$ & \\
\hline NERICA L-47 & $0.008 \%$ & $0.00^{\mathrm{a}}$ & \\
\hline NERICA L-47 & $0.016 \%$ & $0.00^{\mathrm{a}}$ & \\
\hline
\end{tabular}

Mean \pm standard error. Mean of four replicates. NERICA L-47 (0.008 \%); mean of three replicates. NERICA L-47 (0.016 \%); no replicate. Different alphabets in columns indicate significant differences at $5 \%$ level of probability

L-47 (0\%), and NERICA L-47 (0.008\%) did not have a recognizable complementary sequence to the primer OPA02, whereas the primer amplified similar regions of the genome of salinity exposed FARO-44 plants treated $0 \%, 0.004 \%$, and $0.016 \% \mathrm{NaN}_{3}$, respectively. Similarly, no amplifications from primer OPC 04 were reported in

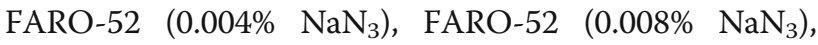
FARO-52 $\left(0.016 \% \mathrm{NaN}_{3}\right)$, FARO-57 $\left(0 \% \mathrm{NaN}_{3}\right)$, NERICA L-47 $\left(0 \% \mathrm{NaN}_{3}\right)$, NERICA L-47 $\left(0.008 \% \mathrm{NaN}_{3}\right)$, and NERICA L-47 (0.004\% $\left.\mathrm{NaN}_{3}\right)$, respectively (Fig. 2). However, similar banding patterns were observed for FARO44 sown under saline conditions but treated with 0 , 0.004 , and $0.16 \% \mathrm{NaN}_{3}$ respectively. Primers OPR 02 (5'-CAC AGC TGC C-3') had type-ability of $52.61 \%$; OPC 04 (5'-CCG CAT CTA C-3') and OPC 05 (5'-
GAT GAC CGC C-3') both had type-ability of $63.16 \%$. Fig. 4 shows germination and screen house experiments while Fig. 5 shows harvested panicles of control and treated plants while Table 7 shows chlorophyll mutations in $\mathrm{M}_{2}$.

\section{Discussion}

Percentage emergence followed the same trends as percentage germination in four of the rice varieties except for NERICA L-47 (Table 2), which was significantly lower than the other varieties. There was no emergence in the highest concentration of sodium azide of NERICA $\mathrm{L}-47$. However, in the second generation $\left(\mathrm{M}_{2}\right)$, percentage emergence was above $80 \%$ for the untreated (control) progenies. Percentage emergence in mutant 
Table 7 Yield parameters of the five varieties

\begin{tabular}{|c|c|c|c|c|c|}
\hline Variety & NaN3 treatment level & Number of tillers/plant at 18 WAP & Number of panicles/ plant at harvest & 100-grain weight (g) & Biomass (g) \\
\hline FARO-44 & Control & $6.00 \pm 1.00^{\mathrm{abcd}}$ & $6.50 \pm 0.50^{\mathrm{abc}}$ & $3.02 \pm 0.06^{\mathrm{ghi}}$ & $8.15 \pm 0.55^{\mathrm{a}}$ \\
\hline FARO-44 & $0 \%$ & $3.75 \pm 0.63^{\mathrm{a}}$ & $4.00 \pm 0.71^{\mathrm{ab}}$ & $2.93 \pm 0.06^{\text {defghi }}$ & $4.98 \pm 1.66^{\mathrm{a}}$ \\
\hline FARO-44 & $0.004 \%$ & $4.50 \pm 0.65^{\mathrm{abc}}$ & $6.50 \pm 1.66^{\mathrm{abc}}$ & $3.03 \pm 0.06^{\mathrm{ghi}}$ & $5.16 \pm 0.48^{\mathrm{a}}$ \\
\hline FARO-44 & $0.008 \%$ & $5.00 \pm 0.41^{\mathrm{abcd}}$ & $5.75 \pm 0.85^{\mathrm{abc}}$ & $3.00 \pm 0.05^{\text {ghi }}$ & $5.92 \pm 0.96^{\mathrm{a}}$ \\
\hline FARO-44 & $0.016 \%$ & $4.75 \pm 0.48^{\mathrm{abcd}}$ & $4.50 \pm 0.96^{\mathrm{ab}}$ & $2.91 \pm 0.08^{\text {cdfghi }}$ & $6.13 \pm 0.61^{\mathrm{a}}$ \\
\hline FARO-44 & $0.032 \%$ & $5.75 \pm 0.75^{\mathrm{abcd}}$ & $6.50 \pm 1.76^{\mathrm{abc}}$ & $3.01 \pm 0.06^{\mathrm{ghi}}$ & $5.72 \pm 0.80^{\mathrm{a}}$ \\
\hline FARO-52 & Control & $10.00 \pm 2.27^{\text {cde }}$ & $8.00 \pm 2.16^{\mathrm{abc}}$ & $2.47 \pm 0.04^{\mathrm{a}}$ & $15.01 \pm 2.75^{\mathrm{abc}}$ \\
\hline FARO-52 & $0 \%$ & $8.00 \pm 0.41^{\mathrm{abcde}}$ & $6.75 \pm 1.11^{\mathrm{abc}}$ & $2.59 \pm 0.06^{\mathrm{abcde}}$ & $15.80 \pm 1.21^{\mathrm{abc}}$ \\
\hline FARO-52 & $0.004 \%$ & $9.00 \pm 2.68^{\mathrm{abcde}}$ & $6.50 \pm 2.10^{\mathrm{abc}}$ & $2.59 \pm 0.11^{\mathrm{abcd}}$ & $15.32 \pm 3.58^{\mathrm{abc}}$ \\
\hline FARO-52 & $0.008 \%$ & $7.5 \pm 0.65^{\text {abcde }}$ & $7.25 \pm 0.48^{\mathrm{abc}}$ & $2.55 \pm 0.07^{\mathrm{ab}}$ & $14.40 \pm 1.91^{\mathrm{abc}}$ \\
\hline FARO-52 & $0.016 \%$ & $6.75 \pm 0.25^{\text {abcde }}$ & $7.00 \pm 0.41^{\mathrm{abc}}$ & $2.52 \pm 0.08^{\mathrm{ab}}$ & $14.08 \pm 0.64^{\mathrm{abc}}$ \\
\hline FARO-52 & $0.032 \%$ & $9.50 \pm 0.87^{\text {bcde }}$ & $8.28 \pm 0.63^{\mathrm{abc}}$ & $2.42 \pm 0.06^{a}$ & $18.02 \pm 2.19^{\mathrm{bc}}$ \\
\hline FARO-57 & Control & $5.25 \pm 0.25^{\mathrm{abcd}}$ & $4.00 \pm 0.41^{\mathrm{ab}}$ & $3.02 \pm 0.13^{\text {ghi }}$ & $9.37 \pm 1.29^{\mathrm{abc}}$ \\
\hline FARO-57 & $0 \%$ & $3.75 \pm 0.49^{a}$ & $3.50 \pm 0.29^{\mathrm{ab}}$ & $3.12 \pm 0.03^{i}$ & $8.59 \pm 0.55^{\mathrm{abc}}$ \\
\hline FARO-57 & $0.004 \%$ & $4.75 \pm 1.18^{\mathrm{abcd}}$ & $5.00 \pm 0.91^{\mathrm{ab}}$ & $3.06 \pm 0.08^{\mathrm{hi}}$ & $11.14 \pm 2.30^{\mathrm{abc}}$ \\
\hline FARO-57 & $0.008 \%$ & $4.50 \pm 0.87^{\mathrm{abc}}$ & $2.75 \pm 0.48^{\mathrm{a}}$ & $2.90 \pm 0.12^{\text {cdfghi }}$ & $9.91 \pm 1.71^{\mathrm{abc}}$ \\
\hline FARO-57 & $0.016 \%$ & $4.25 \pm 1.03^{\mathrm{ab}}$ & $3.75 \pm 0.25^{\mathrm{ab}}$ & $2.77 \pm 0.23^{\text {abcdefghi }}$ & $9.08 \pm 0.54^{\mathrm{abc}}$ \\
\hline FARO-57 & $0.032 \%$ & $5.00 \pm 1.47^{\mathrm{abcd}}$ & $3.75 \pm 0.48^{\mathrm{ab}}$ & $2.93 \pm 0.10^{\mathrm{dfghi}}$ & $11.11 \pm 0.76^{\mathrm{abc}}$ \\
\hline NERICA L-34 & Control & $7.50 \pm 1.04^{\text {abcde }}$ & $7.50 \pm 1.04^{\mathrm{abc}}$ & $2.57 \pm 0.07^{\mathrm{abcd}}$ & $10.07 \pm 2.63^{\mathrm{abc}}$ \\
\hline NERICA L-34 & $0 \%$ & $7.50 \pm 1.44^{\text {abcde }}$ & $3.75 \pm 0.48^{\mathrm{ab}}$ & $2.45 \pm 0.07^{\mathrm{a}}$ & $5.90 \pm 1.08^{\mathrm{a}}$ \\
\hline NERICA L-34 & $0.004 \%$ & $6.50 \pm 0.96^{\text {abcde }}$ & $5.00 \pm 0.71^{a b c}$ & $2.68 \pm 0.03^{\text {abcdefg }}$ & $9.45 \pm 3.82^{\mathrm{abc}}$ \\
\hline NERICA L-34 & $0.008 \%$ & $6.75 \pm 0.48^{\mathrm{abcde}}$ & $6.50 \pm 0.50^{a b c}$ & $2.57 \pm 0.04^{\mathrm{abc}}$ & $6.47 \pm 0.80^{\mathrm{ab}}$ \\
\hline NERICA L-34 & $0.016 \%$ & $8.50 \pm 0.65^{\text {abcde }}$ & $5.50 \pm 0.98^{\mathrm{abc}}$ & $2.68 \pm 0.10^{\mathrm{abcdefg}}$ & $7.91 \pm 1.54^{\mathrm{abc}}$ \\
\hline NERICA L-34 & $0.032 \%$ & $9.25 \pm 0.48^{\text {abcde }}$ & $9.25 \pm 2.21^{\mathrm{abcd}}$ & $2.64 \pm 0.11^{\mathrm{abcdef}}$ & $12.09 \pm 2.31^{\mathrm{abc}}$ \\
\hline NERICA L-47 & Control & $7.00 \pm 0.41^{\mathrm{abcde}}$ & $10.50 \pm 2.53^{\mathrm{bcd}}$ & $2.91 \pm 0.06^{\text {bcdefghi }}$ & $8.61 \pm 2.15^{\mathrm{abc}}$ \\
\hline NERICA L-47 & $0 \%$ & $9.00 \pm 1.87^{\mathrm{abcde}}$ & $6.75 \pm 1.32^{\mathrm{abc}}$ & $2.91 \pm 0.06^{\text {bcdefghi }}$ & $11.56 \pm 3.45^{\mathrm{abc}}$ \\
\hline NERICA L-47 & $0.004 \%$ & $10.25 \pm 1.49^{\mathrm{de}}$ & $12.25 \pm 4.31^{\mathrm{cd}}$ & $2.83 \pm 0.05^{\text {bcdefghi }}$ & $19.20 \pm 6.94^{b c}$ \\
\hline NERICA L-47 & $0.008 \%$ & $12.00 \pm 2.64^{e}$ & $14.00 \pm 2.08^{\mathrm{de}}$ & $3.01 \pm 0.01^{\mathrm{ghi}}$ & $17.45 \pm 2.20^{c}$ \\
\hline NERICA L-47 & $0.016 \%$ & $10.00 \pm 0^{\text {cde }}$ & $17.00 \pm 0^{e}$ & $2.74 \pm 0^{\text {abcdefgh }}$ & $16.62 \pm 0^{\mathrm{abc}}$ \\
\hline
\end{tabular}

Mean \pm standard error. Mean of four replicates. NERICA L-47 (0.008\%); mean of three replicates. NERICA L-47 (0.016\%); no replicate. Different alphabets in columns indicate significant differences at $5 \%$ level of probability

progenies was still significantly lower. It was assumed that there was reduction in seed viability, exacerbated by the inhibition of sodium azide treatment which reduced the chance of survival in the more competitive environment of the soil than in Petri-dishes without having sufficient time to overcome the initial mutagenic inhibition. Mensah and Akomeah (Mensah and Akomeah 1992) have revealed that the higher the mutagenic portion, the lower the tolerance rate, and the present outcomes affirm this previous report. The decline has been ascribed to the physiological aggravation or chromosomal damage imposed on the plant cells by the mutagen. In the present study, percentage germination was not significantly affected by salinity. Similar studies showed that

Table 8 Estimate of parameters of variability, heritability, and genetic gain of some characters of the rice varieties in the $M_{1}$ generation

\begin{tabular}{llllllll}
\hline Characters & Mean & Phenotypic variance $\left(\delta^{2} \mathrm{ph}\right)$ & $\sqrt{ }\left(\delta^{2} \mathrm{ph}\right)$ or $\delta \mathrm{ph}$ & Genotypic variance $\left(\delta^{2} \mathrm{~g}\right)$ & Heritability (\%) & Genetic advance & Genetic gain \\
\hline \% emergence & 77.67 & 169.81 & 13.03 & 79.51 & 46.82 & 12.57 & 16.18 \\
No. of tillers/plant & 6.73 & 20.26 & 4.50 & 15.81 & 78.06 & 7.24 & 107.54 \\
No. of panicles/plant & 6.60 & 36.07 & 6.01 & 28.4 & 78.74 & 9.74 & 147.61 \\
100-grain weight & 2.69 & 1.12 & 1.06 & 1.00 & 98.15 & 2.14 & 79.49 \\
\% lodging & 25.00 & 793 & 28.16 & 203 & 25.60 & 14.85 & 59.40 \\
\hline
\end{tabular}


Table 9 Effect of sodium azide on some parameters of selected progenies in $M_{1}$ and $M_{2}$ generations

\begin{tabular}{|c|c|c|c|c|c|c|}
\hline \multirow[t]{2}{*}{ Progenies from $M_{1}$} & \multirow{2}{*}{$\begin{array}{l}\text { No. of } \\
\text { panicles/ } \\
\text { plant in } M_{1}\end{array}$} & \multicolumn{2}{|c|}{ Percentage emergence } & \multicolumn{2}{|c|}{ Plant heights $(\mathrm{cm})$ at 7 WAP } & \multirow{2}{*}{$\begin{array}{l}\text { Chlorophyll } \\
\text { mutations in } \\
\mathrm{M}_{2}\end{array}$} \\
\hline & & $\overline{M_{1}}$ & $\mathrm{M}_{2}$ & $M_{1}$ & $\mathrm{M}_{2}$ & \\
\hline FARO-44 (control) & $6.50 \pm 1.00$ & $91.25 \pm 4.27$ & $100.00 \pm 0$ & $55.12 \pm 6.46$ & $57.50 \pm 2.25$ & No mutations \\
\hline FARO-44 (0\%) & 11 & $90.00 \pm 1.03$ & $25.00 \pm 2.04$ & $56.75 \pm 2.02$ & $54.50 \pm 2.68$ & No mutations \\
\hline FARO-44 (0.004\% $\left.\mathrm{NaN}_{3}\right)$ & 10 & $92.50 \pm 4.79$ & $20.00 \pm 2.04$ & $58.50 \pm 3.80$ & $52.75 \pm 11.63$ & Striata \\
\hline FARO-44 $\left(0.016 \% \mathrm{NaN}_{3}\right)$ & 9 & $92.50 \pm 4.79$ & $30.00 \pm 3.54$ & $58.50 \pm 3.80$ & $63.13 \pm 3.07$ & Striata \\
\hline FARO-52 (control) & $8.00 \pm 2.16$ & $98.75 \pm 1.25$ & $90.00 \pm 4.08$ & $63.50 \pm 0.87$ & $67.75 \pm 5.07$ & No mutations \\
\hline FARO-52 (0.004\% $\left.\mathrm{NaN}_{3}\right)$ & 10 & $95.00 \pm 5.00$ & $15.00 \pm 2.04$ & $65.25 \pm 2.32$ & $47.33 \pm 10.27$ & Striata \\
\hline FARO-52 (0.008\% $\left.\mathrm{NaN}_{3}\right)$ & 11 & $95.00 \pm 2.89$ & $100.00 \pm 0$ & $55.50 \pm 11.29$ & $55.25 \pm 1.11$ & Viridis \\
\hline FARO-52 $\left(0.016 \% \mathrm{NaN}_{3}\right)$ & 8 & $95.00 \pm 5.00$ & $80.00 \pm 2.04$ & $65.25 \pm 2.32$ & $59.13 \pm 1.61$ & No mutations \\
\hline FARO-57 (control) & $4.00 \pm 0.41$ & $98.75 \pm 1.25$ & $90.00 \pm 3.54$ & $67.00 \pm 3.08$ & $64.25 \pm 1.70$ & No mutations \\
\hline FARO-57 (0\% $\left.\mathrm{NaN}_{3}\right)$ & 7 & $96.25 \pm 2.39$ & $60.00 \pm 5.40$ & $68.00 \pm 1.47$ & $61.38 \pm 2.78$ & No mutations \\
\hline FARO-57 (0.008\% $\left.\mathrm{NaN}_{3}\right)$ & 6 & $96.25 \pm 2.39$ & $60.00 \pm 3.35$ & $68.00 \pm 1.47$ & $59.25 \pm 2.96$ & Striata \\
\hline FARO-57 $\left(0.016 \% \mathrm{NaN}_{3}\right)$ & 5 & $91.25 \pm 4.27$ & $70.00 \pm 3.35$ & $67.00 \pm 1.68$ & $56.75 \pm 1.97$ & Striata \\
\hline NERICA L-34 (control) & $7.50 \pm 1.04$ & $98.75 \pm 1.27$ & $90.00 \pm 2.04$ & $62.75 \pm 2.46$ & $60.13 \pm 0.83$ & No mutations \\
\hline NERICA L-34 $\left(0 \% \mathrm{NaN}_{3}\right)$ & 8 & $92.50 \pm 1.44$ & No emergence & $62.00 \pm 2.61$ & - & - \\
\hline NERICA L-34 (0.004\% NaN $\left.{ }_{3}\right)$ & 14 & $92.50 \pm 1.44$ & $65.00 \pm 5.40$ & $62.00 \pm 2.61$ & $57.25 \pm 1.70$ & Striata \\
\hline NERICA L-34 (0.008\% $\left.\mathrm{NaN}_{3}\right)$ & 12 & $78.75 \pm 5.15$ & $5.00 \pm 0$ & $61.75 \pm 3.59$ & $12.00 \pm 0.72$ & Albino \\
\hline NERICA L-47 (control) & $10.50 \pm 2.53$ & $40.00 \pm 20.92$ & $70.00 \pm 7.36$ & $63.25 \pm 2.63$ & $44.25 \pm 1.32$ & No mutations \\
\hline NERICA L-47 (0\% NaN $\left.{ }_{3}\right)$ & 17 & $1.25 \pm 1.25$ & $5.00 \pm 0$ & $50.00 \pm 0$ & $27.00 \pm 0.87$ & No mutations \\
\hline NERICA L-47(0.004\% $\left.\mathrm{NaN}_{3}\right)$ & 18 & $8.75 \pm 2.39$ & $15.00 \pm 2.04$ & $64.25 \pm 3.07$ & Mutant died at 4 WAP & Albino \\
\hline NERICA L-47 $\left(0.008 \% \mathrm{NaN}_{3}\right)$ & 21 & $8.75 \pm 2.39$ & $5.00 \pm 2.88$ & $64.25 \pm 3.07$ & $47.00 \pm 0.92$ & No mutations \\
\hline NERICA L-47 $\left(0.016 \% \mathrm{NaN}_{3}\right)$ & 13 & $6.25 \pm 2.37$ & No emergence & $56.83 \pm 4.51$ & - & - \\
\hline
\end{tabular}

germination of rice was not affected by salinity (Vwioko and Odigie 2012; Heenan et al. 1988; Khan et al. 1997; Narale et al. 1969).

Mutant plants were shorter in height compared to the control plants in all varieties except NERICA L-47
(Table 3). Sajjad (Sajjad 1984) had previously reported the induction of plants with shorter heights (semi dwarf variants) which was called Basmati rice. Lal et al. (Lal et al. 2009) reported a decrease in seedling heights of Black gram treated with a combination of gamma

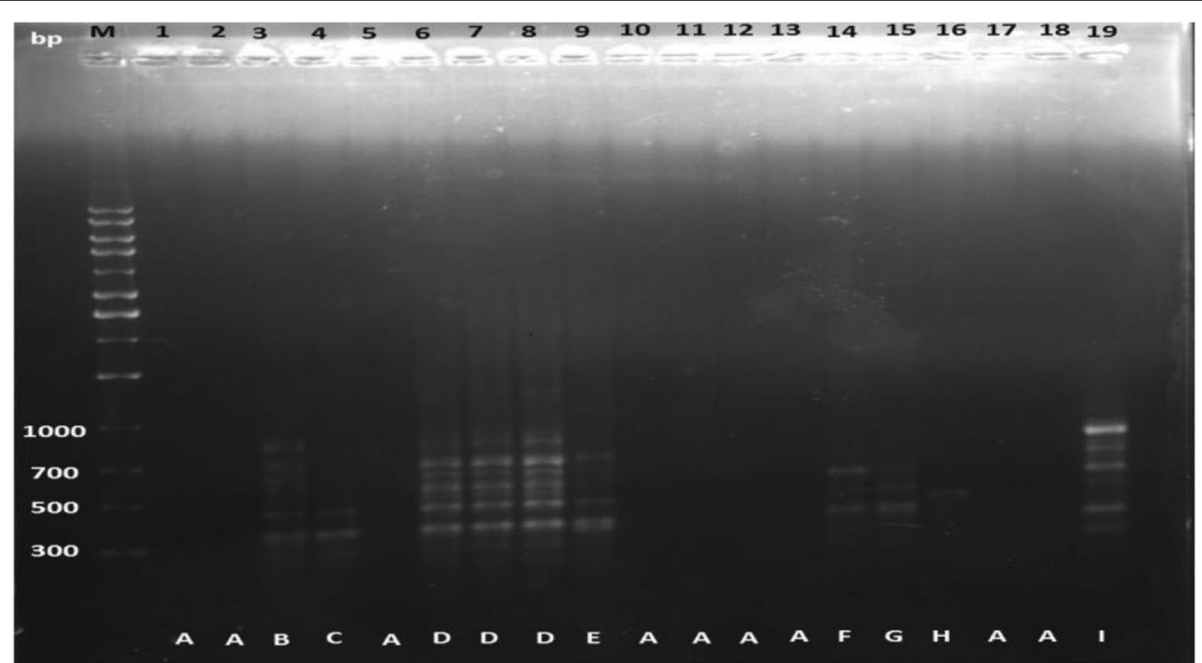

Fig. 1 RAPD agarose gel electrophoresis with OPR 02 (5'-CAC AGC TGC C-3') primer. Same alphabet indicates identical banding pattern. A, Nontype-able DNA; bp, Base pairs; M, 1 kb DNA molecular marker. 1, FARO-44 (control); 2, FARO-52 (control); 3, FARO-57 (control); 4, NERICA L-34 (control); 5, NERICA L-47 (control); 6, FARO-44 (0\% NaN $)$ ); 7, FARO-44 (0.004\% NaN $)$; 8, FARO-44 (0.016\% NaN 3 ); 9, FARO-52 (0.004\% NaN $)$; 10 ,

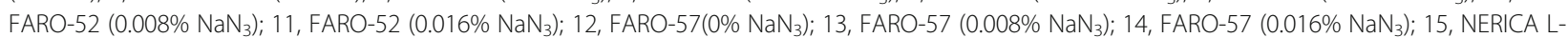

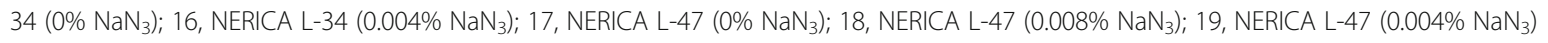




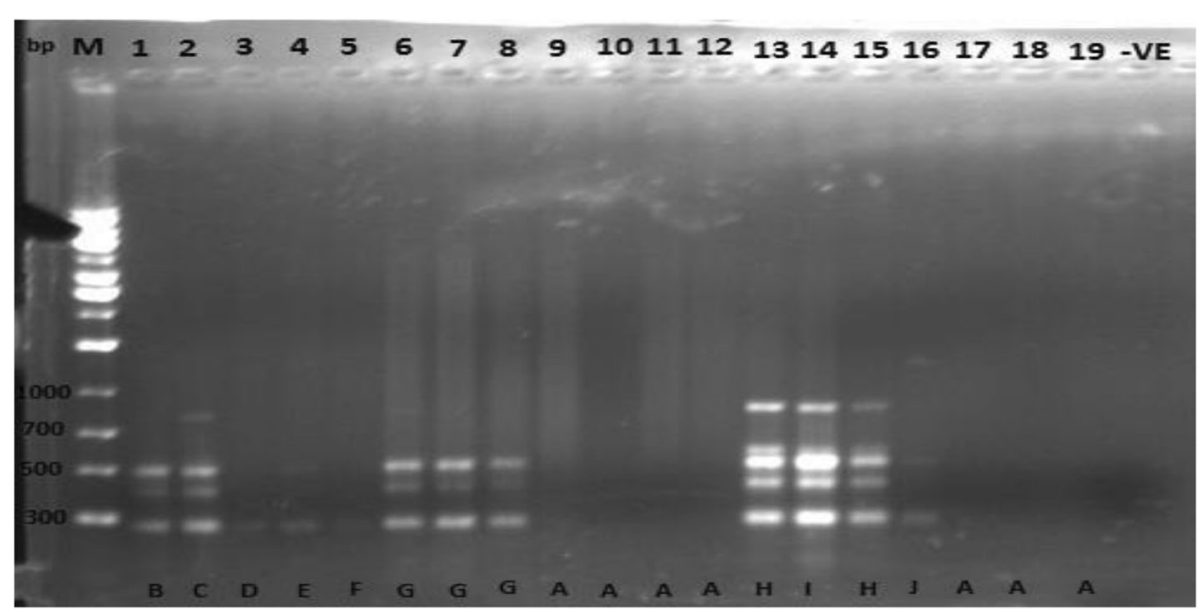

Fig. 2 RAPD agarose gel electrophoresis with OPC 04 (5'-CCG CAT CTA C-3') primer. Same alphabet indicates identical banding pattern. A, Nontype-able DNA; - VE, Negative control; bp, Base pairs; M, 1 kb DNA molecular marker. 1, FARO-44 (control); 2, FARO-52 (control); 3, FARO-57 (control); 4, NERICA L-34 (control); 5, NERICA L-47 (control); 6, FARO-44 (0\% NaN $\mathrm{N}_{3}$; 7, FARO-44 (0.004\% NaN $\left.)_{3}\right) ; 8$, FARO-44 (0.016\% NaN 3 ); 9 , FARO-

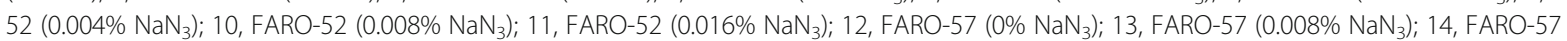
$\left(0.016 \% \mathrm{NaN}_{3}\right) ; 15$, NERICA L-34 (0\% NaN3 $)$; 16, NERICA L-34 (0.004\% NaN $\left.)_{3}\right) ; 17$, NERICA L-47 (0\% NaN $\left.)_{3}\right) ; 18$, NERICA L-47 (0.008\% NaN $)$, 19 , NERICA L-47 $\left(0.004 \% \mathrm{NaN}_{3}\right)$

radiation and sodium azide. However, Mostafa (Mostafa 2011) reported significant increase in heights of Helianthus annus treated with sodium azide. It was found that salinity reduced plant heights in three of the five rice varieties (FARO-44, FARO-57, and NERICA L-34) in relation to control, with the mutant plants taller than the untreated plant exposed to salinity stress. The mean differences however were not significantly different at the eleventh week after planting but had some significance at the seedling stage (week 3). Zeng and Shannon (Zeng and Shannon 2000) stated that reductions in seedlings survival and growth are major causes of stand loss in salt affected fields.

Chlorophyll content index at 5 WAP showed significant differences although there was no particular trend in relation to sodium azide and sodium chloride treatments (Table 4). Mahmoud and Al-Twaty (Mahmoud and Al-Twaty 2006) also reported irregular trends of chlorophyll a, chlorophyll b, and carotenes of tomatoes treated with gamma radiation and sodium azide. Studies

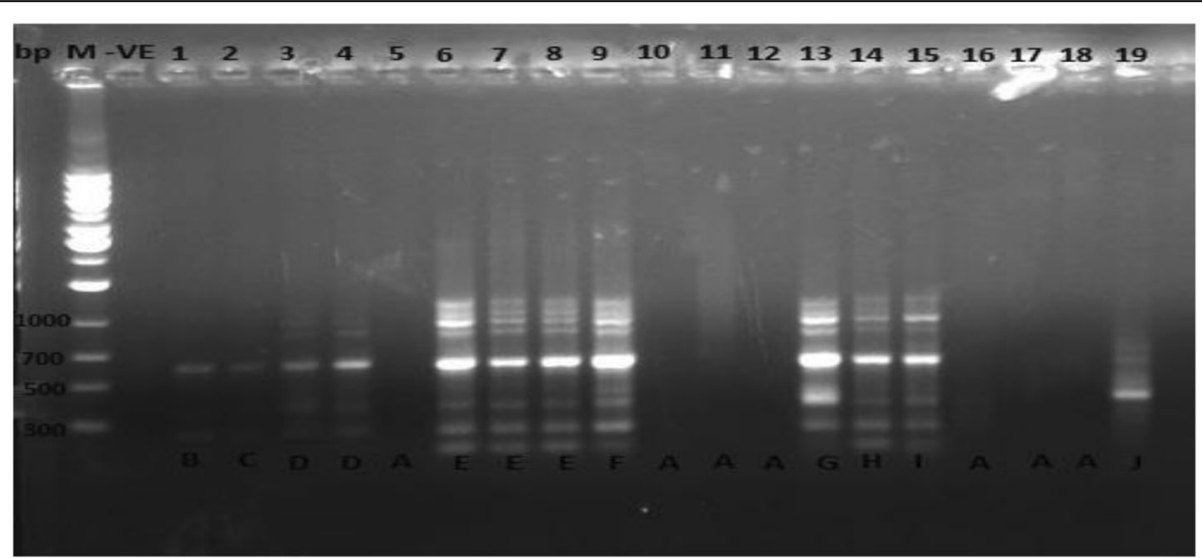

Fig. 3 RAPD agarose gel electrophoresis with OPC 05 (5'-GAT GAC CGC C-3') primer. Same alphabet indicates identical banding pattern. A, Nontype-able DNA; - VE, Negative control; bp, Base pairs; M, 1 kb DNA molecular marker. 1, FARO-44 (control); 2, FARO-52 (control); 3, FARO-57 (control); 4, NERICA L-34 (control); 5, NERICA L-47 (control); 6, FARO-44 (0\% NaN3); 7, FARO-44 (0.004\% NaN $\left.\mathrm{Na}_{3}\right) ; 8$, FARO-44 (0.016\% NaN $\left.)_{3}\right) ; 9$, FARO$52\left(0.004 \% \mathrm{NaN}_{3}\right) ; 10$, FARO-52 (0.008\% NaN 3 ); 11, FARO-52 (0.016\% NaN 3$)_{3}$; 12, FARO-57 (0\% NaN $\left.)_{3}\right) ; 13$, FARO-57 (0.008\% NaN $\left.)_{3}\right) ; 14$, FARO-57 $\left(0.016 \% \mathrm{NaN}_{3}\right) ; 15$, NERICA L-34 $\left(0 \% \mathrm{NaN}_{3}\right) ; 16$, NERICA L-34 (0.004\% NaN $\left.)_{3}\right) ; 17$, NERICA L-47 (0\% NaN $\left.)_{3}\right) ; 18$, NERICA L-47 $\left.(0.008 \% \text { NaN })_{3}\right) ; 19$, NERICA L-47 $\left(0.004 \% \mathrm{NaN}_{3}\right)$ 

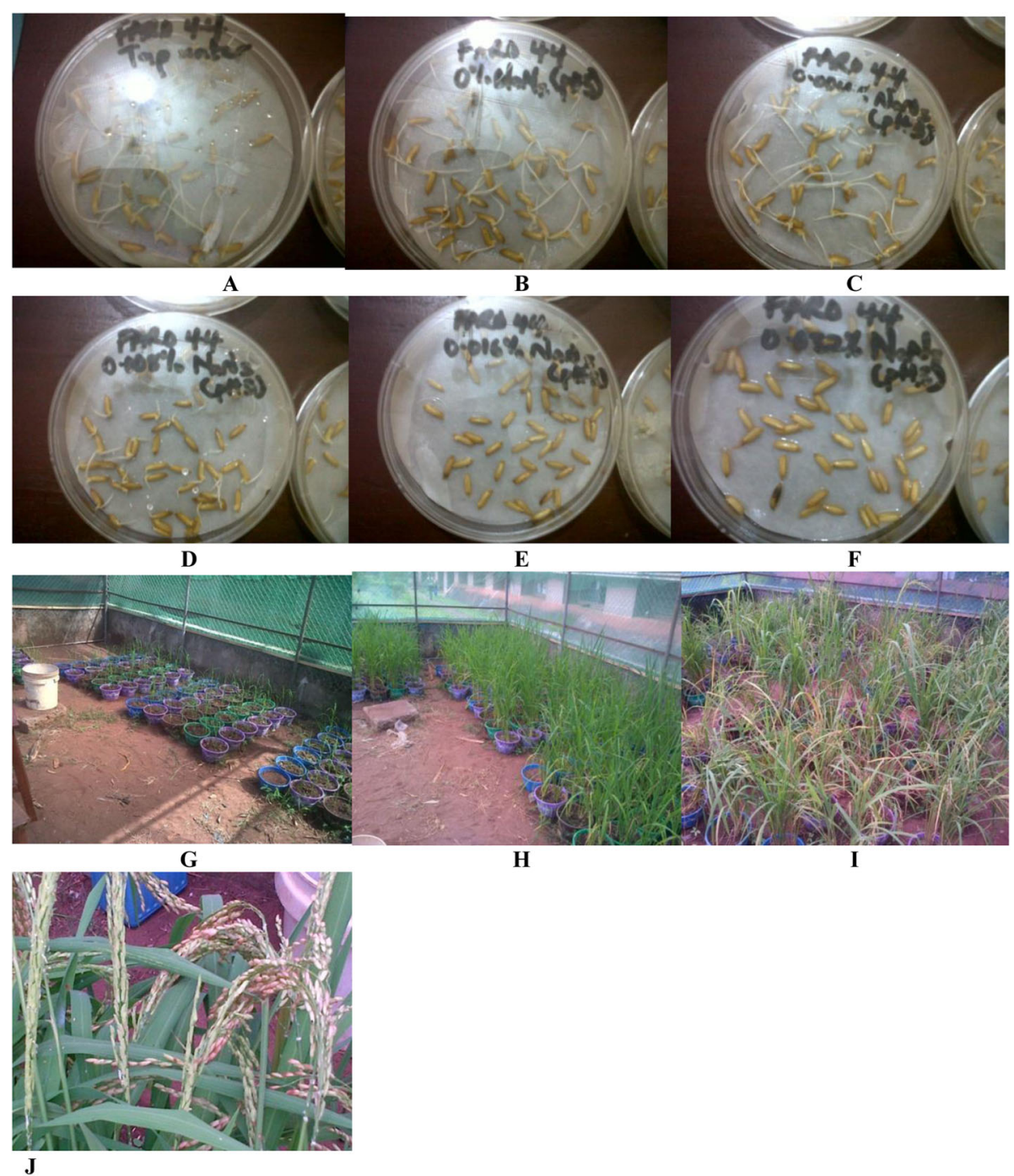

Fig. 4 Germination and screen house experiments. a FARO-44 (control) at 2 DAP. b FARO-44 $\left(0 \% \mathrm{NaN}_{3}\right)$ at 2 DAP. c FARO-44 $(0.004 \%$ NaN 3 ) at 2 DAP. $\mathbf{d}$ FARO-44 $\left(0.008 \% \mathrm{NaN}_{3}\right)$ at 2 DAP. e FARO-44 $\left(0.016 \% \mathrm{NaN}_{3}\right)$ at 2 DAP. f FARO-44 $\left(0.032 \% \mathrm{NaN}_{3}\right)$ at 2 DAP. $\mathbf{g}$ Rice plants at 2 WAP. $\mathbf{h}$ Rice plants at 12 WAP. i Mature rice plants. $\mathbf{j}$ Rice panicles

by Ando and Montalván (Ando and Montalván 2001) revealed that azide treatment was remarkably higher in producing chlorophyll mutations than gamma rays, both on single and combined treatments in rice. However, at 12 WAP, no significant difference was observed in the means of chlorophyll content index in all treatments in relation to their controls in the present studies. Thus, the sodium azide used in the present study as a mutagen had no effect on the chlorophyll apparatus.

The NERICA varieties were the earliest in panicle emergence particularly NERICA L-34 which was earliest irrespective of salt and sodium azide treatments. Closely followed was NERICA L-47; however, sodium azide treatments delayed its flowering (Table 5). FARO-44 and FARO-57 were next to flower. Sodium azide treatment at higher concentrations was observed to stimulate early flowering in FARO-57 whereas FARO-52 was the last to flower. Akinwale et al. (Akinwale et al. 2012) had earlier reported that NERICA L-34 took the shortest time of 105 days to attain $50 \%$ flowering out of twenty rice varieties studied by them. Sodium azide treatment was observed to significantly reduce percentage lodging in the NERICA rice varieties even under salt stress (Table 6). FARO-44 also recorded reductions in percentage lodging under sodium azide treatment. Salinity however did not cause lodging in FARO-44. FARO-52 had the best result with no lodging irrespective of salt or mutagen treatment. FARO-57 had the highest percentage lodging among all the varieties studied, although the highest sodium azide concentration had the lowest value for percentage lodging, mainly as a result of its effect on height. 

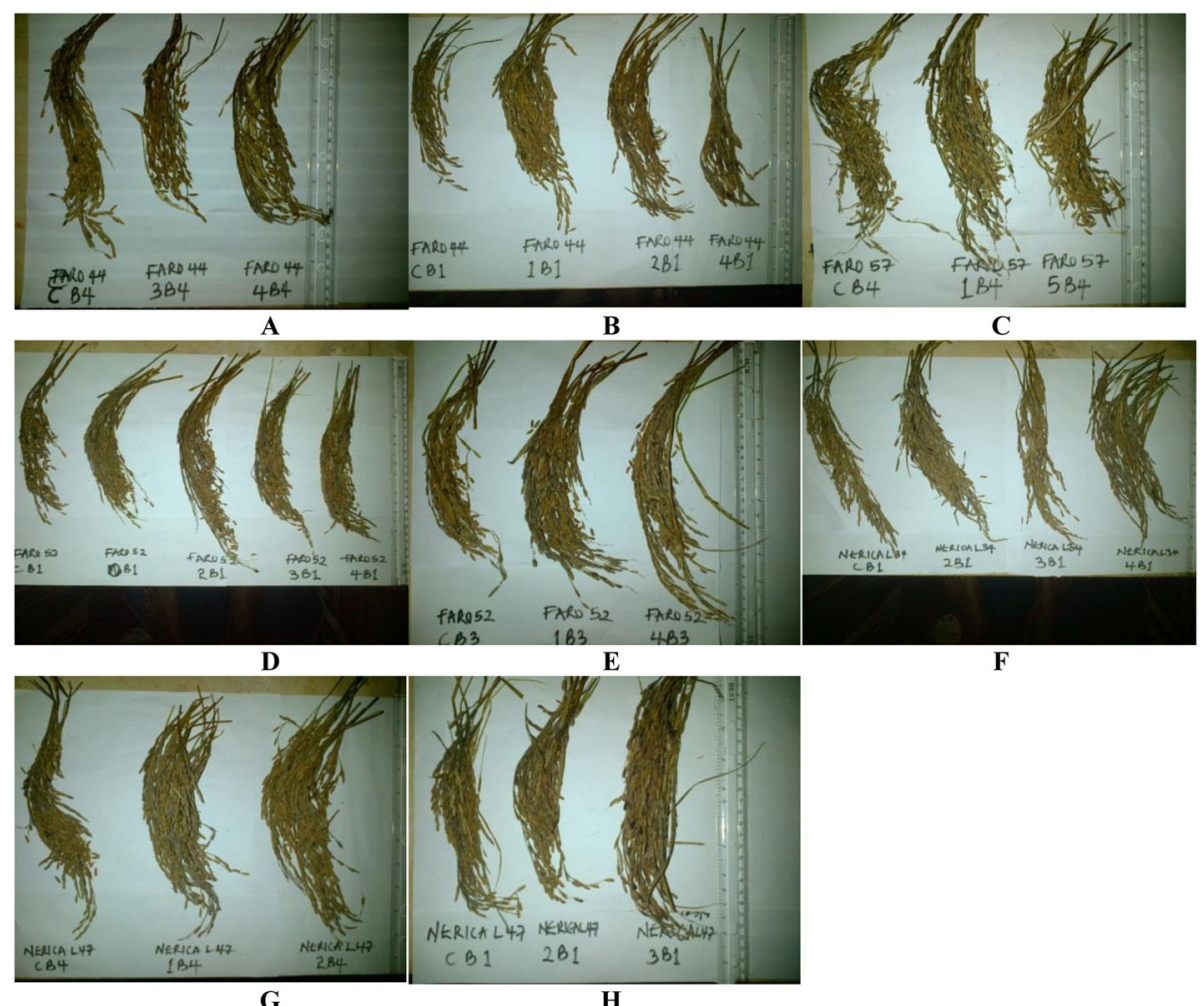

C

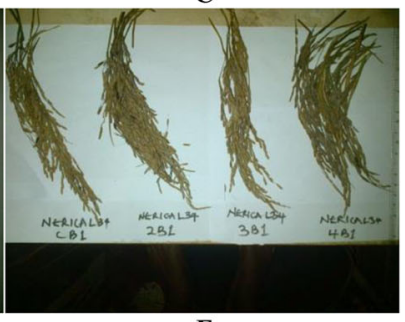

$\mathbf{F}$

Fig. 5 Harvested panicles of control and treated plants. a and $\mathbf{b}$ Comparing panicles of control and $\mathrm{NaN}_{3}$-treated plants in FARO-44. c Comparing panicles of control and NaN3-treated plants in FARO-52. $\mathbf{d}$ and $\mathbf{e}$ Comparing panicles of control and $\mathrm{NaN}_{3}$-treated plants in FARO-57. $\mathbf{f}$ Comparing panicles of control and $\mathrm{NaN}_{3}$ plants in NERICA L-34. $\mathbf{g}$ and $\mathbf{h}$ Comparing panicles of control and NaN $\mathrm{N}_{3}$-treated plants in NERICA L-47

For yield parameters (Table 7), the number of tillers per plant at $18 \mathrm{WAP}$ was negatively affected by salt treatment. However, sodium azide treated plants were less affected even under salt stress. Higher concentrations of sodium azide treatments were observed to progressively increase the number of tillers per plant in all varieties indicating that the chemical reverses the adverse effects of saline conditions. The NERICA varieties treated with sodium azide had more tillers per plant than control even under salt stress. Reports by Vwioko and Odigie (Vwioko and Odigie 2012), Zeng and Shannon (Zeng and Shannon 2000), and Doberman and Fairhurst (Doberman and Fairhurst 2000) showed that salinity significantly reduced number of tillers in rice. (Sajjad 1984) reported that rice plants subjected to gamma rays were superior in productive tillers per plant than their untreated parent plant.

Salt stress also reduced the number of panicles per plant in all varieties. However, the number of panicles per plant was significantly increased in sodium azide treated plants under salt stress especially in the NERICAs with significantly higher number of panicles per plant than their controls. Shereen et al. (Shereen et al. 2005) mentioned that decrease in panicle numbers, panicle length, and sterility were the significant reasons for loss of yield. Studies by (Khan et al. 2004) showed that the numbers of pods were increased with sodium azide treatment in Mung bean.

The 100-grain weight showed significant variations across the varieties as there was no particular trend irrespective of salt or sodium azide treatment.

Biomass of all varieties, except FARO-52, was significantly reduced by sodium chloride treatment. The biomass, however, of sodium azide treated plants under salt stress was significantly higher than their controls. Biomass was increased with increasing doses of sodium azide treated FARO-57 and the NERICA varieties.

For the genetic parameters of variation, heritability, and hereditary improvement for five chosen parameters, genetic variation (GV) went from 1.06 to 203.00. The phenotypic variance (PV) went from 1.12 to 793.00 (Table 8). The phenotypic variance was higher than the genetic variance. Be that as it may, the contrasts between the two estimations were low for number of tillers per plant, number of panicles per plant, and 100-grain weight surmising low ecological impact on these attributes. The distinctions were higher for percentage sprouting and percentage lodging. Heritability was 78.06\% for number of tillers per plant, $78.74 \%$ for number of panicles per plant, and $98.15 \%$ for 100 -grain. 
Mensah and Obadoni (Mensah and Obadoni 2007) revealed that there were increments in parameters, for example, coefficient of variation, heritability, and genetic addition of yield parameters in groundnut under sodium azide treatment, showing the chance of advancing better return variations through selection. They additionally expressed that heritability communicates the unwavering quality of the phenotypic value as a manual for breeding. Khan et al. (Khan et al. 2004) demonstrated that mutagenic medicines modified mean values and made extra genetic variability for quantitative attributes in mungbean.

The results of these previous studies agree with the results of the present research.

Chlorophyll mutations observed within the spectrum considered in $\mathrm{M}_{2}$ were albino, striata, and viridis, of which the striata type was predominant (Table 9, Fig. 6). This result almost agrees with Ando and Montalván (Ando and Montalván 2001) who observed that within the spectrum and frequency of the chlorophyll mutations; the albino type of mutations was predominant in physical and chemical mutagen-treated populations in rice followed by the viridis type. Mensah and Obadoni (Mensah and Obadoni 2007) reported that out of xantha, chlorina, and albino chlorophyll mutants considered in assessing the mutation frequency, xantha type was predominant in two cultivars of groundnut treated with sodium azide. Lal et al. (Lal et al. 2009) observed that of the six different types of chlorophyll mutants, namely, albino, xantha, dark xantha, chlorina, viridis, and striata, induced xantha and dark xantha were most frequent while striata was least frequent.

RAPD analysis with all three primers used revealed different DNA banding patterns between control plants and the sodium azide treated progenies (Figs 1, 2, and 3 ). This implies that the genetic constitution of the parent varieties has been altered by the mutagenic treatments. This agrees with the studies of other researchers that have shown that PCR based RAPD can detect changes in DNA brought about by induced mutation (Anil Kumar et al. 2012; Atak et al. 2011; Barakat and El-Sammak 2011; Behera et al. 2012; Hamzekhanlu et al. 2011; Taryono and Human 2011; Yaycili and Alikamanoğlu 2012).

\section{Conclusions}

The observations from the present research indicate that sodium azide is a potent mutagen. This is evident from information on genetic variability, heritability as well as results recorded at the molecular level by the DNA profiles of the rice varieties and their mutant counterparts. The mutagenic effect of the sodium azide on the rice

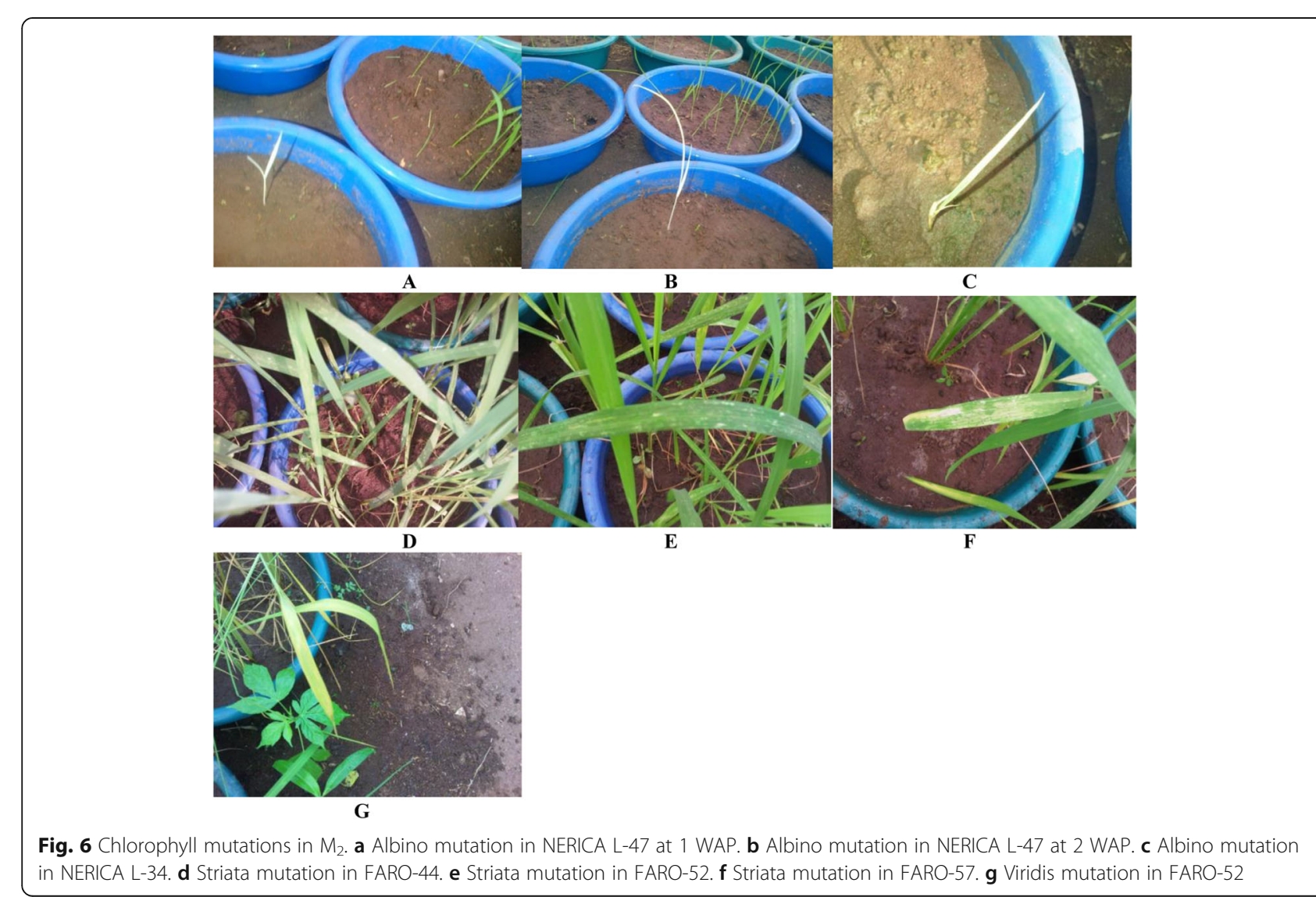


varieties has conferred characteristics on the plants that will enable them to strive better than their parent plants without compromising yield and particularly in areas with salt challenges. However, further screening on promising mutant plants from this research should be done through successive generations to identify stable traits and variant that can perform optimally under saline conditions.

\section{Abbreviations \\ CCI: Chlorophyll content index; DAP: Days after planting; IRRI: International Rice Research Institute; $L_{50}$ : Amount of toxic agent that is capable of killing $50 \%$ of plant population; $\mathrm{NaCl}$ : Sodium chloride; $\mathrm{NaN}_{3}$ : Sodium azide; PCR: Polymerase Chain Reaction; RAPD: Random amplified polymorphic DNA; SSA: Sub-Saharan Africa; THC: Total hydrocarbon content; WAP: Weeks after planting}

\section{Acknowledgements}

The researchers are grateful to the Department of Plant Biology and Biotechnology, University of Benin, Nigeria, for the facilities. The mentorship and efforts of Prof. Joseph Kwesi Mensah of the Department of Botany, Ambrose Alli University, Ekpoma, Nigeria, during the course of the study is very much appreciated.

\section{Authors' contributions}

$\mathrm{BI}$ and UEO designed and carried out the study. BI and UEO carried out the statistical analyses and interpretation of the study. BI and UEO wrote the first draft of the manuscript. BI wrote the final draft of manuscript. The authors read and approved the final manuscript.

\section{Funding}

Funding for the study was partly provided by the UniBen-URPC Funding 2014.

\section{Availability of data and materials}

Please contact author for data requests.

\section{Ethics approval and consent to participate}

Not applicable

\section{Consent for publication}

Consent is given for publication of this manuscript when accepted.

\section{Competing interests}

The authors declare no conflicts of interests.

Received: 26 March 2020 Accepted: 21 May 2020

Published online: 05 June 2020

\section{References}

Abdullah Z, Ahmed R (1982) Salt tolerance of Solanum tuberosum L. growing on saline soils amended with gypsum. Z Acker und Pftanzenbau 151:409-416

Adamu AK, Aliyu H (2007) Morphological effects of sodium azide on tomatoes (Lycopersicumesculentum Mill). Sci World J 2:9-12

Ahloowalia BS, Maluszynski M (2001) Induced mutation: a new paradigm in plant breeding. Euphytica. 118:167-173

Akinwale MG, Akinyele BO, Odiyi AC, Nwilene F, Gregorio G, Oyetunji OE. Phenotypic screening of Nigerian rain-fed lowland mega rice varieties for submergence tolerance. Proceedings of the world congress on engineering.4 - 6 July, London. 2012; p6.

Akita S, Cabuslay GS. Physiological basis of differential response to salinity in rice cultivars. In: Proceedings of the third International Symposium in Genetic Aspects of Plant Mineral Nutrition. 19 - 24 June, Braunschwing, German.1988; $37 p$.

Alika JE. Statistics and research methods. 2ndedn. Ambik Press, Benin City, Nigeria. 2006; p369.

Allard RW (1999) Principles of plant breeding. John Wiley and Sons, New York, p 264
Ando A, Montalván R (2001) Gamma-ray radiation and sodium azide $\left(\mathrm{NaN}_{3}\right)$ mutagenic efficiency in rice. Crop Breeding and Applied Biotechnology 1: $339-346$

Anil Kumar HV, Muralidhar TS, Munirajappa S (2012) RAPD analysis of EMS mutagenised mulberry genotype RFS135. Scholarly Journal of Biotechnology $1: 1-7$

AOAC (Association of Official Analytical Chemists). Official methods of analysis of AOAC international (18thed). AOAC International, Rockville, MD. 2005.

Apogee Instruments Incorporated. How it works. http://www.apogeeinstruments. com. Accessed 26 June 2007.

Atak C, Celik O, Leyla AL (2011) Genetic analysis of Rhododendron mutants using random amplified polymorphic DNA (RAPD). Pak J Bot 43:1173-1182

Barakat MN, El-Sammak H (2011) In vitro mutagenesis, plant regeneration and characterization of mutants via RAPD analysis in baby's breathGypsophila paniculata L. Aust J Crop Sci 5:214-222

Behera M, Panigrahi J, Mishra RR, Rath SP (2012) Analysis of EMS induced in in vitro mutants of Asteracanthanlongifolia (L.) Nees using RAPD markers. Indian J Biotechnol 11:39-47

Bertagne-Sagnard B, Fouilloux G, Chupeau Y (1996) Induced albino mutations as a tool for genetic analysis and cell biology in flax (Linumusitatssimum). J Exp Biol 47:189-194

Bhat TA, Khan AH, Parveen S (2005) Comparative analysis of meiotic abnormalities induced by gamma rays, EMS and MMS in Viciafaba L. Journal of Indian Botanical Society 84:45-58

Coe EH, Neuffer MG (1977) The genetics of corn. In: Spragues GF (ed) Corn and corn improvement. American Society of Agronomy, Madison, Wisconsin., pp $111-213$

Crayford GW, Shen C (1998) The origin of rice agriculture: recent progress in East Asia. Antiquity. 72:858-866

Dalton TJ, Guei RG (2003) Productivity gains from rice genetic enhancements in West Africa: countries and ecologies. World Dev 31:359-374

Dhanayanth KPM, Reddy V (2000) Cytogenetic effects of gamma rays and ethylmethanesulphonate in chilli piper (Capsicum annum). Cytologia. 65:129-133

Doberman A, Fairhurst T. Rice nutrient disorders and nutrient management. Handbook Series. Potash and Phosphate Institute of Canada (PPIC) and International Rice Research Institute, Canada. 2000; p191.

Ebert G, Botella J, Ali-Dinar H, Ladder P (2002) Ameliorating effects of $\mathrm{Ca}\left(\mathrm{NO}_{3}\right)_{2}$ on growth, mineral uptake and photosynthesis of $\mathrm{NaCl}$ - stress guava seedling (Psidiumguajava L.). Sci Hortic 93:125-135

El-Sharabasy HM, Ibrahim A (2010) Communities of Oribatid mites and heavy metal accumulation in Oribatid species in agricultural soils in Egypt impacted by wastewater. Plant Prot Sci 46:159-170

Grant WF, Salamone MF (1994) Comparative mutagenecity of chemicals selected for test in the international program on chemical safety collaborative study on plant systems for the detection of environmental mutagens. Mutat Res 30:187-209

Guei RG, Fagade SO. Promising technologies for rice production in West and Central Africa. West Africa Rice Development Association (WARDA), Bouake. 2002; p 28.

Gustafson A. The mutation system of the chlorophyll apparatus. CWK Gleerup, Lund, Sweden. 1940; p 40.

Hamzekhanlu M, Izadi-Darbandi A, Pirvali-Beiranvand N, Taher-Hallajian M, Majdabad A (2011) Phenotypic and molecular analysis of $M_{7}$ generation of soybean mutant lines through random amplified polymorphic DNA (RAPD) marker and some morphological traits. Afr J Agric Res 6:1779-1785

Heenan DP, Lewin LG, McCaffery DW (1988) Salinity tolerance in rice varieties at different growth stages. Aust J Exp Agric 28:343-349

Heong KL, Chen YH, Johnson DE, Jahn GC, Hossain M, Hamilton RS (2005) Debate over genetic modified rice trial in China. Sci Lett 310:231-233

Ikhajiagbe B, Akendolor A (2016) Comparative effects of pretreatment of stem cuttings of Chromolaenaodorata (Siam weed) with sodium azide and hydroxylamide on the survival and phytoremediative performance in an oilpolluted soil. Nigeria Journal of Biotechnology 30(1):27-39

Ikhajiagbe B, Anoliefo GO. Changes in metals and polyaromatic hydrocarbon contents of a spentlubrication oil-polluted soil after exposure to sodium azide and hydroxlamine hydrochloride solutions: implications for intrinsic bioremediation. Studia Universitatis Babeş - Bolyai, Biologia. 2017; LXII, 1: $57-66$.

Ikhajiagbe B, Oshomoh EO (2014) Influence of sodium azide on the germination success of Vignaunquiculata (TVU-3541 accession) exposed to water soluble fractions of waste engine oil. Journal of Laboratory Science 2(1):26-30 
Ikhajiagbe B, Mgbeze GC, Erhenhi HA (2009) Growth and yield responses of Sphenostylisstenocarpa (Hochst ex A. Rich) Hams to phosphate enrichment of soil. Afr J Biotechnol 8:641-643

Ikhajiagbe B, Odigie EU, Okoh BE, Agho EE (2013) Effects of sodium azide on the survival, growth and yield performance of rice (Oryza sativa, FARO-57 variety) in a hydrocarbon-polluted soil. The International Journal of Biotechnology 2(1):28-41

International Rice Research Institute. Global rice shortage sparks panic - SBC World, New Australia. 2008.

Johnson HW, Robinson HF, Comstock RE (1955) Estimation of genetic and environmental variability in soybean. Agron J 47:314-318

Juliano BO, Bechtel DB. The rice grain and its gross composition. In: Juliano BO (ed) Rice chemistry and technology. America association of cereal chemistry. St. Paul, Minnesota. 1985; pp 619-645

Khan MSA, Hamid A, Karim MA (1997) Effect of sodium chloride on germination and seedling characters of different types of rice (Oryza sativa L.). J Agron Crop Sci 179:163-169

Khan S, Wani MR, Parveen K (2004) Induced genetic variability for quantitative traits in VignaRadiata(L.) Wilczek. Pak J Bot 36:845-850

Lal GM, Toms B, Lal SS (2009) Induced chlorophyll mutations in black gram. Asian Journal of Agricultural Sciences 1:1-3

Lauchli A, Epstein E (1990) Plant responses to saline and sodic conditions. In: Tanji KK (ed) Agricultural salinity assessment and management. ASCE, New York., pp 113-137

Lutts S, Kinet JM, Bouharmont J (1995) Changes in plant response to $\mathrm{NaCl}$ during development of rice (Oryza sativa L.) varieties differing in salinity resistance. J Exp Bot 46:1843-1852

Mahmoud AA, Al-Twaty N (2006) Effect of gamma irradiation and sodium azide on some economic traits in tomato. Saudi Journal of Biological Sciences 13: 44-49

Maji AT. He knows what he wants. In:Bentley J, Mele PV, Mohapatra S (eds) Rice to feed africa: stories from the emergency rice initiative project workshop. Africa rice center (AfricaRice) Cotonou, Benin. 2009; p5.

Mashenkov A (1986) Induced mutation process in a source of new mutants. Maize Genetics Cooperation Newsletter 60:70-71

Mensah JK, Akomeah PA (1992) Mutagenetic effects of hydroxylamine and streptomycin on the growth and yield of cowpea Vignaunguiculata (L.)Walp. Legume. Research. 15:39-44

Mensah JK, Obadoni B (2007) Effects of sodium azide on yield parameters of groundnut (ArachishypogaeaL.). Afr J Biotechnol 6:668-671

Mensah JK, Akomeah PA, Ikhajiagbe B, Ekpekurede EO (2006) Effects of salinity on germination, growth and yield of five groundnut genotypes. Afr J Biotechnol 5:1973-1979

Mostafa GG (2011) Effects of sodium azide on the growth and variability induction in Helianthus annus L. International Journal of Plant Breeding and Genetics 5:76-85

Mshembula BP, Mensah JK, Ikhajiagbe B (2012) Comparative assessment of the mutagenic effects of sodium azide on some selected growth and yield parameters of five accessions of cowpea - Tvu-3615, Tvu-2521, Tvu-3541, Tvu-3485 and Tvu-3574. Archives of Applied Science Research 4(4):1682-1691

Narale RP, Subramangan TK, Mukherjee RK (1969) Influence of salinity on germination, vegetative growth and grain yield of rice (Oryza sativa var. Dular). Agron J 61:341-343

Nelson DW, Sommers LE. Total carbon, organic carbon and organic matter. In: Methods of soil analysis part II. ASA/SSSA, Madison WI. 1982; pp539-579.

Nwanze KF, Mohapatra S, Kormawa P, Keya S, Bruce O (2006) Perspective: rice development in sub-Saharan Africa. J Sci Food Agric 86:675-677

Nwilene FE, Oikeh SO, Agunbiade TA, Oladimeji O, Ajayi O, Sié M, Gregorio GB, Togola A, Touré AD. Growing lowland rice: a production handbook. Africa rice center (WARDA) Cotonou, Benin. 2008; p40.

Ojehomon VET, Adebayo SB, Ogundele OO, Okoruwa VO, Ajayi O, Diagne A, Ogunlana O. Rice data systems in Nigeria (National rice survey 2009): building a rice data system for sub-Saharan Africa. ICE data systems in Nigeria. 2009; p61.

Omoregie UE, Mensah JK, Ikhajiagbe B (2012) Germination response of five varieties treated with sodium azide. Research Journal of Mutagenesis 4(1):1422

Oputa CO (1981) Response of Celosia argenteaL. to salinity, morphological changes and ionic adjustments. Nigerian Journal of Science 3:45-53

Osuji LC, Nwoye I (2007) An appraisal on the impact of petroleum hydrocarbon on soil fertility: the Owaza experience. Afr J Agric Res 2:318-324
Owais WM, Kleinhofs A (1988) Metabolic activation of the mutagen azide in biological systems. Mutat Res 197:313-323

Owais WM, Rosichan JL, Ronald RC, Kleinhofs A, Nillian RA (1983) A mutagenic metabolite synthesized in the presence of azide is azidoalanine. Mutat Res 118:229-239

Raicu P, Mixich F (1992) Cytological effects of sodium encapsulated in liposomes on the heteropliodes cell cultures. Mutat Res 283:215-219

Rao GM, Roa VM (1983) Mutagenic efficiency, effectiveness and factor of effectiveness of physical and chemical mutagen in rice. Cytologia. 48:427436

Rines HW (1995) Sodium azide mutagenesis in diploid and hexaploid oats and comparison with ethylmethanesulphonate treatments. Environ Exp Bot 25:7-

Sajjad MS (1984) Induction of semi-dwarf mutants in basmati rice (Oryza sativa L. cv BAS .370). Philippine Journal of Crop Science 9:75-77

Shannon MC, Rhoades JD, Draper JH, Scardaci SC, Spyres MD (1998) Assessment of salt tolerance in rice cultivars in response to salinity problems in California. Crop Sci 38:394-398

Shereen A, Mumtaz S, Raza S, Khan MA, Solangi S (2005) Salinity effects on seedling growth and yield components of different inbred rice lines. Pak J Bot 37:131-139

Singh BN, Fagade S, Nkwungwu MN, Williams C, Jagtap SS, Oladimeji O, Efisue A, Okhidievbie O (1997) Rice growing environments and biophysical constraints in different agroecological zones of Nigeria. Meteorological Journal 2:35-44

Tanji KK (1990) Nature and extent of agricultural salinity. In: Tanji KK (ed) Agricultural salinity assessment and management. ASCE, New York., pp 1-13

Tara JL, Dnyansager VR (1980) Comparison of ethylemethanesulphonate and induced radiation induced abnormalities in Turneraulmifolial. var. angustitifolia wild. Cytologia 56:221-231

Taryono CP, Human S (2011) The detection of mutational changes in Sorghum using RAPD. Indian J Biotechnol 16:66-70

Van der Veen JH. Arabidopsis.Information Service.1966; 3:26.

Van RW, Den-Bulk HJM, Loffer WH, Lindhout KM (1990) Somaclonal variation tomato: effect of explants source and a comparison with chemical mutagenesis. Theoretical Applied Genetics 80:817-825

Veleminsky J, Anglis KJ (1987) Effect of sodium azide on replicative and repair DNA synthesis in barley embryos. Mutat Res 190:125-129

Vwioko ED, Odigie UE (2012) Assessment of growth performance of FARO-44 rice cultivar in salt - treated soil. University of Benin Journal of Science and Technology 1:20-29

Windmeijer PN, Duivenbooden NV, Andriesse W. Characterization of rice growing agro-ecosystems in west Africa: semi - detailed characterization of inland valleys in Cote d' Ivoire. Wageningen agricultural university, Wageningen.1994; p72.

Yaycili O, Alikamanoğlu S (2012) Induction of salt-tolerant potato (Solanum tuberosumL.) mutants with gamma irradiation and characterization of genetic variations via RAPD-PCR analysis. Turk J Biol 36:405-412

Yeo AR, Yeo ME, Flowers SA, Flowers TJ (1990) Screening of rice (Oryza sativa L.) genotypes for physiological characters contributing to salinity resistance and their relationship to overall performance. Theoretical Applied Genetics 79: 377-384

Zeerak NA (1991) Cytogenetical effects of gamma rays and ethylmethanesulphonate in brinjal (Solanum melogena L.). Cytologia 56:639643

Zeng L, Shannon MC (2000) Salinity effects on the seedling growth and yield components of rice. Crop Sci 40:996-1003

Zhang H, Hodson J (2001) Engineering salt tolerant Brassica plants: characterization of yield and seed oil quality in transgenic plants with Increase vacuolar sodium accumulation. Proc Natl Acad Sci 48:12832-12836

\section{Publisher's Note}

Springer Nature remains neutral with regard to jurisdictional claims in published maps and institutional affiliations. 